\title{
ON SOME LIMIT THEOREMS FOR CONTINUED FRACTIONS
}

\author{
JORGE D. SAMUR
}

\begin{abstract}
As a consequence of previous results on mixing random variables, some functional limit theorems for quantities related to the continued fraction expansion of a random number in $(0,1)$ are given.
\end{abstract}

\section{INTRODUCTION}

The aim of this paper is to collect some results about the convergence in distribution of sums of some random variables associated to the continued fraction expansion of a random number $\omega$ in $(0,1)$.

As discussed in $\S 2$, the results in $[24,26]$ apply directly to the sequence $\left\{a_{j}\right\}$ of partial quotients when $\omega$ is chosen under Gauss's measure. If it is replaced by any probability measure absolutely continuous with respect to Lebesgue measure, similar results hold (by [20, Lemma 1]; in the case of Lebesgue measure [12, Lemma 19.4.2] works). Then some theorems of Lévy [18, 19] and Doeblin [7] are obtained as corollaries and some information is added (see Examples 2.6, 2.14 and Remarks 2.7, 2.15 for references). In particular, we get necessary and sufficient conditions on a function $f$ for the validity of a functional limit theorem (invariance principle) for sums $\sum_{j \leq n} f\left(a_{j}\right)$ under Lebesgue measure on $(0,1)$; then a certain class of positive functions $f$ of real argument is examined and we obtain (Corollaries 2.12 and 2.13) functional limit theorems for $f$ regularly varying (and bounded on finite intervals).

In $\S 4$ we consider sums involving $x_{j}$, the complete quotients, and $u_{j}$, defined in (4.1), which measure the approximation of $\omega$ by its convergents. We extend some results of $\S 2$ (see Examples 4.1) including functional limit theorems for $\sum_{j \leq n} f\left(x_{j}\right)$ and $\sum_{j \leq n} f\left(u_{j}\right)$ for some regularly varying $f$; in the case of $\left\{x_{j}\right\}$, Corollary 4.2 generalizes [18, Theorem 4] and Corollary 4.6 contains for a certain class of regularly varying functions a result suggested in $[18, \mathrm{pp} .200-$ 201]. Example 4.7.2 gives the functional form of a limit theorem indicated by Doeblin. Lemma 4.5, which is used to deal with $u_{j}$, essentially contains

Received by the editors December 2, 1987.

1980 Mathematics Subject Classification (1985 Revision). Primary 60F05, 60F17, 11K50; Secondary $60 \mathrm{~B} 12,11 \mathrm{~K} 60,26 \mathrm{~A} 12$.

Key words and phrases. Continued fraction expansion, mixing random variables, functional limit theorem, invariance principle, regularly varying function, approximation by convergents.

Some results were announced at the 5th International Conference on Probability in Banach spaces, Medford, Massachusetts, July 1984. 
the theorem in [15]; the proof given here is based on a relation due to Lévy (Proposition 2.1).

In order to achieve these extensions of the results of $\S 2$, we isolate from [5] (and [11]) some facts which lead to Corollary 3.4 (see Remark 4.4(a)).

\section{SUMS OF FUNCTIONS OF THE PARTIAL QUOTIENTS}

Given an irrational number $\alpha \in(0,1)$, let

$$
\alpha=\left[0, a_{1}(\alpha), a_{2}(\alpha), \ldots\right]
$$

be its (infinite) simple continued fraction expansion, defined by the continued fraction algorithm

$$
\begin{gathered}
\alpha=\frac{1}{x_{1}(\alpha)}, \quad x_{1}(\alpha)=a_{1}(\alpha)+\frac{1}{x_{2}(\alpha)}, \ldots, \\
x_{n}(\alpha)=a_{n}(\alpha)+\frac{1}{x_{n+1}(\alpha)}, \ldots
\end{gathered}
$$

where $a_{n}(\alpha)=\left[x_{n}(\alpha)\right]$ (throughout the paper, [.] denotes the integer part of a real number; we refer to [4, $\$ 4$ ] and [10] or [17] for the elementary facts about continued fractions). The $a_{n}$ 's are the partial quotients and the $x_{n}$ 's the complete quotients of $\alpha$.

We are interested in $a_{j}$ and $x_{j}$ as functions defined on the set of irrational numbers in $(0,1)$. Denote it by $\Omega$ and let $\mathscr{B}$ be the class of its Borel subsets. On $(\Omega, \mathscr{B})$ we will consider the Lebesgue measure $\lambda$ and Gauss's measure

$$
P(B)=\frac{1}{\log 2} \int_{B} \frac{d \omega}{1+\omega}, \quad B \in \mathscr{B} .
$$

If $\rho$ is a probability measure on $(\Omega, \mathscr{B})$ we shall write $E_{\rho}$ (similarly $\operatorname{Var}_{\rho}, \operatorname{Cov}_{\rho}$ ) for the corresponding expectation operator and $\mathscr{L}_{\rho}(\xi)$ for the law of a random element $\xi$ defined on $(\Omega, \mathscr{B}, \rho)$; often we will write $E=E_{P}$, $\mathscr{L}=\mathscr{L}_{P}$. If moreover $\rho$ is absolutely continuous with respect to $\lambda$ we shall write $\rho \ll \lambda$.

Also we will deal with the functions $p_{n}, q_{n}$ defined for $\omega \in \Omega$ by

$$
\begin{gathered}
p_{0}(\omega)=0, p_{1}(\omega)=1, p_{n}(\omega)=a_{n}(\omega) p_{n-1}(\omega)+p_{n-2}(\omega) \quad \text { if } n \geq 2, \\
q_{0}(\omega)=1, q_{1}(\omega)=a_{1}(\omega), q_{n}(\omega)=a_{n}(\omega) q_{n-1}(\omega)+q_{n-2}(\omega) \quad \text { if } n \geq 2 .
\end{gathered}
$$

For each $\omega \in \Omega$ and $n \geq 0, p_{n}(\omega) / q_{n}(\omega)=\left[0, a_{1}(\omega), \ldots, a_{n}(\omega)\right]$ is the $n t h$ convergent to $\omega$.

Following Lévy [20, Chapitre IX] we write, for $n \geq 1$ and $\omega \in \Omega$

$$
y_{n}(\omega):=\frac{q_{n}(\omega)}{q_{n-1}(\omega)}=\left[a_{n}(\omega), a_{n-1}(\omega), \ldots, a_{1}(\omega)\right] .
$$

It is well known that endowing $(\Omega, \mathscr{B})$ with Gauss's measure $P,\left\{a_{j}: j \geq 1\right\}$ is a (strictly) stationary and $\psi$-mixing sequence of r.v.'s with an exponential 
mixing rate and satisfies the condition $\psi^{*}<\infty([4$, p. 50$]$ or [12]; the last fact follows from the right inequality in (4.15) of [4]).

Throughout the paper, we use freely notation and concepts quoted in [24]. The dependence coefficients $\phi(k), \psi(k), \psi^{*}$ refer to $\left\{a_{j}\right\}$ defined on $(\Omega, \mathscr{B}, P)$.

The following relation, due to Lévy [19, equality (8) in $\S 74]$ and called the Borel-Lévy formula by Doeblin [7], will be useful later (the indicated dependence properties of $\left\{a_{j}\right\}$ can be proved starting from it [19]).

2.1. Proposition. If $n \geq 2, y=\left[k_{n-1}, \ldots, k_{1}\right]$ with $k_{1}, \ldots, k_{n-1} \in \mathbf{N}^{*}$ and $1 \leq a<b$ then

$$
\lambda\left(a<x_{n} \leq b \mid y_{n-1}=y\right)=\lambda((a, b]) \frac{y(y+1)}{(y a+1)(y b+1)} .
$$

(Apart from being stated here in $\Omega$, this is (4.12) of [4] since

$$
T^{n-1}=x_{n}^{-1} \text { if } T \omega=\omega^{-1}-\left[\omega^{-1}\right]
$$

and

$$
\begin{aligned}
\left\{\omega \in \Omega: y_{n-1}(\omega)\right. & \left.=\left[k_{n-1}, \ldots, k_{1}\right]\right\} \\
& \left.=\left\{\omega \in \Omega: a_{1}(\omega)=k_{1}, \ldots, a_{n-1}(\omega)=k_{n-1}\right\} .\right)
\end{aligned}
$$

In order to apply some of the results in $[24,26]$ it appears to be necessary to verify that $\phi(1)<1$ and this can be done using Proposition 2.1. But, under the properties of $\left\{a_{j}\right\}$ indicated above, no further argument is needed. The following property was overlooked by us in $[24,26]$ and is stated by Bradley in [6, p. 184]: given a probability space $(X, \mathscr{A}, Q)$ and two sub- $\sigma$-algebras $\mathscr{M}$, $\mathscr{N}$ of $\mathscr{A}$ we have $\phi:=\phi(\mathscr{M}, \mathscr{N})<1$ if $\psi^{*}:=\psi^{*}(\mathscr{M}, \mathscr{N})<\infty$ (see for example [26] for the definitions). For the sake of completeness, we show that $\phi \leq 1-\left(\psi^{*}\right)^{-1}$ if $\psi^{*}<\infty \quad\left(\psi^{*} \geq 1\right.$ always $)$. Assume $\phi>0$; observe that for each $\varepsilon \in(0, \phi)$ there exist $A \in \mathscr{M}, B \in \mathscr{N}$ such that $Q(A)>0$ and

$$
\phi-\varepsilon<(Q(A B)-Q(A) Q(B)) / Q(A) \leq 1-Q(B)
$$

(if $Q(A B)-Q(A) Q(B)<0, Q\left(A B^{c}\right)-Q(A) Q\left(B^{c}\right)=-(Q(A B)-Q(A) Q(B))>$ $0)$ which implies $Q(B)>0$ and

$$
(1-(\phi-\varepsilon))^{-1}(\phi-\varepsilon)<Q(B)^{-1}(\phi-\varepsilon)<\psi^{*}-1 .
$$

The inequality follows from this. We remark that in a recent article Philipp [23] proves the stronger fact that $\psi(1)<0.8$ for $\left\{a_{j}\right\}$, thus obtaining $\phi(1)<0.4$.

In this section, $H$ denotes a real separable Hilbert space with norm $\|\cdot\|$ and inner product $\langle\cdot, \cdot\rangle$. For the sake of clarity, we recall some facts and terminology about certain measures on (the Borel $\sigma$-algebra of) $H$ (see [2]). If $\nu$ is an infinitely divisible (i.d.) probability measure then there exist a symmetric nonnegative trace class operator $S$ and a Lévy measure $\mu$ (in the Hilbert space case, it can be described as a nonnegative measure which satisfies

$$
\left.\int \min \left\{1,\|x\|^{2}\right\} \mu(d x)<\infty\right)
$$


such that for each $\tau>0$ there exists $z_{\tau} \in H$ such that the characteristic functional of $\nu$ can be written as

$$
\begin{aligned}
\hat{\nu}(y)=\exp \left\{i\left\langle z_{\tau}, y\right\rangle\right. & -\frac{1}{2}\langle S y, y\rangle \\
& \left.+\int\left(e^{i\langle x, y\rangle}-1-i\langle x, y\rangle I_{B_{\tau}}(x)\right) \mu(d x)\right\}
\end{aligned}
$$

$\left(y \in H ; B_{\tau}=\{x:\|x\| \leq \tau\}\right) . \quad S$ and $\mu$ are uniquely determined by $\nu$ and so is $z_{\tau}$ for each $\tau$. When $z_{\tau}$ and $\mu$ vanish, $\nu$ is the centered Gaussian measure with covariance operator $S$ (with the notation of [24], we have $\left.\Phi_{\nu}(\langle\cdot, y\rangle,\langle\cdot, y\rangle)=\int\langle x, y\rangle^{2} \nu(d x)=\langle S y, y\rangle\right)$. If $z_{\tau}$ and $S$ are zero, $\nu$ is called the $\tau$-centered Poisson measure with Lévy measure $\mu$ and is denoted by $c_{\tau}$ Pois $\mu$; if $\mu$ is finite, $c_{\tau}$ Pois $\mu=($ Pois $\mu) * \delta_{b_{\tau}}$ where $b_{\tau}=-\int_{B_{\tau}} x \mu(d x)$ and Pois $\mu$ is $\exp (-\mu(H)) \exp (\mu)$, whose characteristic functional is $\exp (\hat{\mu}-\mu(H))$; the classical Poisson measure with parameter $\lambda>0$ is $\operatorname{Pois}\left(\lambda \delta_{1}\right)$. Therefore, if $\nu$ is i.d. relation (2.1) says that, for each $\tau>0$,

$$
\nu=\delta_{z_{\tau}} * \gamma * c_{\tau} \text { Pois } \mu
$$

$\gamma$ being the centered Gaussian measure with covariance operator $S$; this is the Lévy-Khintchine representation of $\nu$.

The Skorohod space (see [5]) of $H$-valued functions on $[0,1]$ shall be denoted by $D([0,1], H)$ and we shall write $D=D([0,1], \mathbf{R})$. If $\nu$ is an i.d. probability measure on $H, Q_{\nu}$ denotes the law on $D([0,1], H)$ of a stochastic process $\xi=\{\xi(t): t \in[0,1]\}$ with stationary independent increments, trajectories in $D([0,1], H), \xi(0)=0$ and $\xi(1)$ having law $\nu$.

If $\left\{X_{n j}\right\}=\left\{X_{n j}: j=1, \ldots, n, n \geq 1\right\}$ is a double array of $H$-valued measurable functions on $(\Omega, \mathscr{B})$ we shall consider the property

$$
\left\{r_{n}\right\} \subset \mathbf{N}^{*}, r_{n} \leq n, r_{n} / n \rightarrow 0 \Rightarrow \sum_{j=1}^{r_{n}} X_{n j} \rightarrow 0 \text { in measure. }
$$

In our first statements we refer directly to some assertions in [24], taking there $B=H, j_{n}=n, \mathscr{L}=\mathscr{L}_{p}, E=E_{P}$ and replacing the letter $f$ by $h$ to denote functionals.

2.2. Proposition. Let $\left\{f_{n}: n \geq 1\right\}$ be a sequence of functions from $\mathbf{N}^{*}$ into $H$ and define $X_{n j}=f_{n}\left(a_{j}\right)$ if $j=1, \ldots, n, n \geq 1$. Suppose that the following conditions of [24, Corollary 6.5] are satisfied: (1), (2) modified by assuming the existence of the limits only for $h$ in a sequentially $w^{*}$-dense subset $W$ of $H^{\prime}$, (3). Then (a) and (b) of that result hold and

(c) for any $\rho \ll \lambda$ and for every $\tau \in C(\mu)$,

$$
\mathscr{L}_{\rho}\left(\xi_{n}^{(\tau)}\right) \rightarrow_{\omega} Q_{\gamma^{*}{ }^{*}{ }_{\tau} \text { Pois } \mu} \text { in } D([0,1], H)
$$

where

$$
\xi_{n}^{(\tau)}(t)=\sum_{1 \leq j \leq[n t]}\left(X_{n j}-E_{P} X_{n 1 \tau}\right) \quad(t \in[0,1])
$$


Proof. Use [24, Corollary 6.5, 26, Corollary 3.3(iii)] and Lemma 2.3 below, noting that $\left\{X_{n j}-E X_{n 1 \tau}\right\}$ satisfies $(*)$ (see the proof of [24, Corollary 6.5].

2.3. Lemma. Let $\rho \ll \lambda$. Assume $\left\{f_{n}\right\},\left\{X_{n j}\right\}$ are as in Proposition 2.2, $\left\{X_{n j}\right\}$ satisfying $(*)$.

(a) Let $\xi_{n}(t)=\sum_{1 \leq j \leq[n t]} X_{n j}(t \in[0,1])$. If $\left\{\mathscr{L}_{P}\left(\xi_{n}\right)\right\}$ or $\left\{\mathscr{L}_{\rho}\left(\xi_{n}\right)\right\}$ converges weakly (in $D([0,1], H))$ then both sequences have the same limit.

(b) Part (a) holds with $\sum_{j=1}^{n} X_{n j}$ in place of $\xi_{n}$.

Proof. (a) Take $\left\{r_{n}\right\}$ as in the definition of $(*)$ with $r_{n} \rightarrow \infty$; write $\tilde{\xi}_{n}(t)=$ $\sum_{r_{n}<j \leq[n t]} X_{n j}(t \in[0,1])$. First we observe that

$$
\sup _{t \in[0,1]}\left\|\xi_{n}(t)-\tilde{\xi}_{n}(t)\right\|=\max _{k \leq r_{n}}\left\|\sum_{j=1}^{k} X_{n j}\right\| \rightarrow 0 \text { in measure }
$$

(this follows from $(*)$ and a well-known maximal inequality quoted, for example, in [24, Proposition 2.2]).

On the other hand, if $g$ is any bounded continuous real function on $D([0,1], H)$, Lemma 1 of [20] shows that $\lim _{n}\left(E_{P} g\left(\tilde{\xi}_{n}\right)-E_{\rho} g\left(\tilde{\xi}_{n}\right)\right)=0$ since $\tilde{\xi}_{n}$ is $\sigma\left(a_{j} ; j>r_{n}\right)$-measurable.

2.4 Proposition. Let $\left\{f_{n}\right\}$ and $\left\{X_{n j}\right\}$ be as in Proposition 2.2. Suppose that for some $\rho \ll \lambda,\left\{\mathscr{L}_{\rho}\left(\sum_{j=1}^{n} X_{n j}\right)\right\}$ converges weakly to a probability measure $\nu$ on $H$.

(I) If $\left\{X_{n j}\right\}$ satisfies $(*)$ then $\nu$ is i.d. and if (2.2), $\tau \in C(\mu)$, is its LévyKhintchine representation, assertions (a)-(c) of [24, Theorem 6.2] hold and also we have $\left(\mathrm{b}^{\prime}\right)$ of $[24$, Corollary 6.3] if the second part of (ii) of that result is satisfied.

(II) Let $\xi_{n}$ be the random function $\xi_{n}(t)=\sum_{1 \leq j \leq[n t]} X_{n j}(t \in[0,1])$ and suppose that $\left\{\mathscr{L}_{\lambda}\left(\xi_{n}\right)\right\}$ is relatively compact in $D([0,1], H)$. Then $\left\{X_{n j}\right\}$ satisfies $(*), \nu$ is $i . d$. and $\mathscr{L}_{\rho}\left(\xi_{n}\right) \rightarrow{ }_{w} Q_{\nu}$.

Proof. (I) Lemma 2.3 and [24].

(II) The argument in [26, Theorem 3.2, proof of (III) $\Rightarrow$ (II)] shows that $\left\{X_{n j}\right\}$ satisfies $(*)$. Then use (I), Lemma 2.3 and [26, Theorem 3.2].

2.5. Remark. In the real-valued case, the convergence in law of $\xi_{n}^{(\tau)}(1)$ in Proposition 2.2 also follows from the main theorem in [16], which improves [3]; it gives necessary and sufficient conditions (under certain preliminary assumptions) even in the nonstationary case. See [26, Remark 3.4.2] for another reference (convergence to stable laws).

Next we give examples which are related to some results in [7].

\subsection{Examples.}

2.6.1. Let $l^{2}$ be the Hilbert space of square summable real sequences and let $\left\{e_{p}: p \geq 1\right\}$ be its canonical orthonormal basis. Define $\Gamma^{(n)}: \Omega \rightarrow l^{2}$ by

$$
\Gamma_{p}^{(n)}(\omega)=\operatorname{card}\left\{j \leq n: a_{j}(\omega)=p\right\}, \quad p \geq 1, \omega \in \Omega,
$$


and $\gamma=\left(\gamma_{p}\right)_{p \geq 1}$ by

$$
\gamma_{p}=P\left(a_{1}=p\right)=\frac{1}{\log 2} \log \left(1+\frac{1}{p(p+2)}\right), \quad p \geq 1 .
$$

Then, if $\xi_{n}$ is the random function

$$
\xi_{n}(t)=n^{-1 / 2}\left(\Gamma^{([n t])}-[n t] \gamma\right) \quad(t \in[0,1]),
$$

for any $\rho \ll \lambda$ we have $\mathscr{L}_{\rho}\left(\xi_{n}\right) \rightarrow_{w} Q_{\nu}$, where $\nu$ is the centered Gaussian measure on $l^{2}$ whose covariance operator $S$ satisfies

$\left\langle S e_{p}, e_{q}\right\rangle=\delta_{p q} \gamma_{p}-\gamma_{p} \gamma_{q}+2 \sum_{j=1}^{\infty}\left\{P\left(a_{1}=p, a_{j+1}=q\right)-\gamma_{p} \gamma_{q}\right\}, \quad p \geq 1, q \geq 1$;

here $\delta_{p q}=1$ if $p=q$, $=0$ if $p \neq q$.

Proof. Let $f(p)=e_{p}$ and take $f_{n}(p)=n^{-1 / 2}(f(p)-\gamma)$. Since $E_{P}\left\|f\left(a_{1}\right)\right\|^{2}<$ $\infty$, by the same arguments which led from [24, Corollary 4.5] to [24, Corollary 4.7] we can verify that $\left\{f_{n}\right\}$ satisfies the hypotheses of Proposition 2.2 with $\mu=0$ and $\Phi(h)=\operatorname{Var}_{P} h\left(a_{1}\right)+2 \sum_{j=1}^{\infty} \operatorname{Cov}_{P}\left(h\left(a_{1}\right), h\left(a_{j+1}\right)\right.$ ) (see also [24, Remark on p. 405]). Concerning (2.4), we remark that $P\left(a_{1}=p, a_{j+1}=q\right)=$ $P\left(a_{1}=q, a_{j+1}=p\right)$ (see $[18$, p. 182]).

2.6.2. Let $\theta>0$ and $\alpha \in \mathbf{R}$. For each $n \geq 1$ define $\xi_{n}$ by

$$
\xi_{n}(t)=n^{-\alpha} \sum_{1 \leq j \leq[n t]} a_{j}^{\alpha} I_{\left\{a_{j}>\theta n\right\}} \quad(t \in[0,1]) .
$$

Then for any $\rho \ll \lambda, \mathscr{L}_{\rho}\left(\xi_{n}\right) \rightarrow{ }_{w} Q_{\nu}$ where

(a) if $\alpha>0, \nu=$ Pois $\mu$ with $\mu(d x)=I_{\left(\theta^{\alpha}, \infty\right)}(x)(\alpha \log 2)^{-1} x^{-1 / \alpha-1} d x$, i.e., the characteristic function of $\nu$ is

$$
\hat{\nu}(y)=\exp \left\{(\alpha \log 2)^{-1}\left(\int_{\theta^{\alpha}}^{\infty} e^{i x y} x^{-1 / \alpha-1} d x-\alpha \theta^{-1}\right)\right\}
$$

(b) if $\alpha<0, \nu=$ Pois $\mu$ with $\mu(d x)=I_{\left(0, \theta^{*}\right)}(x)(-\alpha \log 2)^{-1} x^{-1 / \alpha-1} d x$, i.e.,

$$
\hat{\nu}(y)=\exp \left\{(-\alpha \log 2)^{-1}\left(\int_{0}^{\theta^{\prime \prime}} e^{i x y} x^{-1 / \alpha-1} d x+\alpha \theta^{-1}\right)\right\}
$$

(c) if $\alpha=0$, then $\xi_{n}(t)=\operatorname{card}\left\{j \leq[n t]: a_{j}>\theta n\right\},(t \in[0,1])$ and $\nu=$ $\operatorname{Pois}\left((\theta \log 2)^{-1} \delta_{1}\right)$.

Proof. Take $f_{n}(p)=(p / n)^{\alpha} I_{(\theta n, \infty)}(p)$ in Proposition 2.2. Condition (1) there is satisfied with the corresponding $\mu$ because, for positive $x$,

$$
P\left(a_{1}>x\right)=\frac{1}{\log 2} \log \left(1+\frac{1}{[x]+1}\right) \sim \frac{1}{\log 2} \cdot \frac{1}{x} \quad \text { as } x \rightarrow \infty .
$$


On the other hand, observe that if $\alpha>0, X_{n 1 \delta}=0$ for $\delta \in\left(0, \theta^{\alpha}\right]$. For (b), note that $\sup _{n} n E X_{n 1 \delta}^{2} \leq \delta^{2} \sup _{n} n P\left(a_{1}>\theta n\right)=O\left(\delta^{2}\right)$ and that $\lim _{n} n E X_{n 1 \theta^{n}}=\left((1-\alpha) \theta^{1-\alpha} \log 2\right)^{-1}$. If $\alpha=0$ then $X_{n 1 \delta}=0$ for $\delta \in$ $(0,1)$.

2.6.3. Fix a sequence $\left\{\theta_{r}\right\}$ such that $0<\theta_{1}<\theta_{2}<\cdots$ and $\lim _{r} \theta_{r}=\infty$. Define $L^{(n)}: \Omega \rightarrow l^{2}$ by

$$
L_{r}^{(n)}(\omega)=\operatorname{card}\left\{j \leq n: \theta_{r} n<a_{j}(\omega) \leq \theta_{r+1} n\right\}, \quad r \geq 1, \omega \in \Omega,
$$

and $\xi_{n}$ by $\xi_{n}(t)=L^{([n t])}, t \in[0,1]$. Then for any $\rho \ll \lambda, \mathscr{L}_{\rho}\left(\xi_{n}\right) \rightarrow_{w} Q_{\nu}$ where

$$
\nu=\text { Pois } \mu \quad \text { with } \mu=\sum_{r=1}^{\infty} \frac{1}{\log 2}\left(\frac{1}{\theta_{r}}-\frac{1}{\theta_{r+1}}\right) \delta_{e_{r}} .
$$

Moreover, $(\operatorname{Pois} \mu)(F)=1$ where $F=\left\{\left(x_{1}, x_{2}, \ldots\right) \in l^{2}: x_{r} \in \mathbf{N}\right.$ and only a finite number of $x_{r}$ 's is nonzero $\}$ and

$$
(\text { Pois } \mu)(\{x\})=\exp \left(-\frac{1}{\theta_{1} \log 2}\right) \prod_{r \geq 1} \frac{1}{x_{r} !}\left(\frac{1}{\log 2}\left(\frac{1}{\theta_{r}}-\frac{1}{\theta_{r+1}}\right)\right)^{x_{r}} \quad \text { if } x \in F .
$$

Proof. Take $f_{n}(p)=\sum_{r=1}^{\infty} I_{\left(\theta_{r} n, \theta_{r+1} n\right]}(p) e_{r}$ in Proposition 2.2. Note that for every $\delta \in(0,1), X_{n 1 \delta}=0$ and that for any subset $A$ of $H$ we have

$\left|\left(n \mathscr{L}\left(X_{n 1}\right) \mid B_{\delta}^{c}\right)(A)-\mu(A)\right| \leq \sum_{r=1}^{\infty}\left|n P\left(\theta_{r} n<a_{1} \leq \theta_{r+1} n\right)-(\log 2)^{-1}\left(\theta_{r}^{-1}-\theta_{r+1}^{-1}\right)\right|$

which goes to zero as $n \rightarrow \infty$ because each term tends to zero and

$$
\begin{aligned}
\sum_{r=1}^{\infty} n P\left(\theta_{r} n<a_{1} \leq \theta_{r+1} n\right) & =n P\left(a_{1}>\theta_{1} n\right) \rightarrow\left(\theta_{1} \log 2\right)^{-1} \\
& =\sum_{r=1}^{\infty}(\log 2)^{-1}\left(\theta_{r}^{-1}-\theta_{r+1}^{-1}\right) .
\end{aligned}
$$

The expression for Pois $\mu$ follows by direct calculation of $\mu^{* n}, n \geq 1$.

2.7. Remarks. Example 2.6 .1 gives a natural extension of the result in $[7, \S 2$, no. 5]. The limit laws of $\xi_{n}(1)$ given in (a) and (c) of 2.6 .2 appear in [7, §4, §3] where (a), case $\alpha=1$, is used for deriving the limit law of $\xi_{n}(1)$ in Example 2.14.2 below. The proofs presented in [7] of both results have been objected and the last one established in [13] by using [8].

Now we are interested in sums of the form $\sum_{j \leq n} f\left(a_{j}\right)$.

2.8. Proposition. Let $f$ be a function from $\mathbf{N}^{*}$ into $\mathbf{R}$ and let $\{x(n)\} \subset$ $\mathbf{R}$ and $\{b(n)\} \subset(0, \infty)$ with $b(n) \rightarrow \infty$. Assume that for some $\rho \ll \lambda$, 
$L_{\rho}\left(b(n)^{-1}\left(\sum_{1}^{n} f\left(a_{j}\right)-n x(n)\right)\right) \underset{w}{\rightarrow} \nu$, a nondegenerate probability measure. Then $\nu$ is stable.

Proof. Since $b(n) \rightarrow \infty$ we can find $\left\{r_{n}\right\} \subset \mathbf{N}^{*}, r_{n} \leq n, r_{n} \rightarrow \infty$ such that $b(n)^{-1} \sum_{1}^{r_{n}} f\left(a_{j}\right) \rightarrow 0$ in measure. Arguing as in the proof of Lemma 2.3 we can replace $\rho$ by $P$ in our hypothesis and then [26, Remark 3.4.3.1] or [22, Theorem 2] concludes the proof.

A function $R:[r, \infty) \rightarrow(0, \infty) \quad(r>0)$ is regularly varying $($ at $\infty)$ with exponent $\alpha \in \mathbf{R}[27,2]$ if it is Borel measurable and $\lim _{x \rightarrow \infty} R(t x)(R(x))^{-1}=$ $t^{\prime *}$ for every $t>0$. If $\alpha=0, R$ is slowly varying.

2.9. Proposition. Let $f: \mathbf{N}^{*} \rightarrow \mathbf{R}$.

(a) Let $\{x(n)\} \subset \mathbf{R}$ and $\{b(n)\} \subset(0, \infty)$ with $b(n) \rightarrow \infty$. The following assertions are equivalent:

(I) The random functions $\xi_{n}$ defined by

$$
\xi_{n}(t)=b(n)^{-1} \sum_{1 \leq j \leq[n t]}\left(f\left(a_{j}\right)-x(n)\right) \quad(t \in[0,1])
$$

satisfy

$$
\mathscr{L}_{\lambda}\left(\xi_{n}\right) \underset{w}{\rightarrow} W, \quad \text { the Wiener measure on } D .
$$

(II) $\mathscr{L}_{\lambda}\left(b(n)^{-1} \sum_{1}^{n}\left(f\left(a_{j}\right)-x(n)\right)\right) \rightarrow_{w} N(0,1)$, the standard normal distribution, and $\left\{X_{n j}\right\}:=\left\{b(n)^{-1}\left(f\left(a_{j}\right)-x(n)\right): 1 \leq j \leq n, n \geq 1\right\}$ satisfies $(*)$.

(b) The assertion

(A) there exist a bounded sequence $\{x(n)\} \subset \mathbf{R}$ and $\{b(n)\} \subset(0, \infty)$ with $b(n) \rightarrow \infty$ such that (I) is satisfied,

holds if and only if

$$
\lim _{x \rightarrow \infty} \frac{x^{2} \sum_{k:|f(k)|>x} k^{-2}}{\sum_{k:|f(k)| \leq x} f^{2}(k) k^{-2}}=0
$$

or, equivalently, if

$$
U(x):=(\log 2)^{-1} \sum_{k:|f(k)| \leq x} f^{2}(k) k^{-2}
$$

is slowly varying. If this is the case and $U(x) \rightarrow \infty$ as $x \rightarrow \infty$, we can take $x(n)=E_{P} f\left(a_{1}\right)$ and any $\{b(n)\}$ such that $\lim _{n} n b(n)^{-2} U(b(n))=1$.

(c) If (I) holds for some $\{x(n)\},\{b(n)\}$, then (2.7) holds with $\lambda$ replaced by any $\rho \ll \lambda$.

Proof. (a) and (c): [26, Corollary 3.3(iii)] and Lemma 2.3.

(b) First we observe that $U^{\prime}(x):=E_{P}\left(f^{2}\left(a_{1}\right) ;\left|f\left(a_{1}\right)\right| \leq x\right)$ is slowly varying if and only if $U$ is ( $U$ and $U^{\prime}$ both have a finite limit as $x \rightarrow \infty$ or both tend 
to $\infty$; in the later case, $U \sim U^{\prime}$ by (2.3)); moreover this holds if and only if (2.8) is satisfied.

Then, that (2.8) implies (I) (with $\{x(n)\}$ and $\{b(n)\}$ as indicated in the case $U(x) \rightarrow \infty$ ) follows from [26, Corollary 3.7] and its proof (see [25]) noting that, with the notation there, $\Phi_{1}^{(0)}=1$ and $\Phi_{j}^{(0)}=0$ if $j \geq 2$ (use that $\psi^{*}<\infty$ and [24, Proposition 2.7]).

For the converse, suppose that (II) holds and that $U^{\prime}(x) \rightarrow \infty$ as $x \rightarrow$ $\infty$. Fix $\delta \in(0,1)$ and write $Y_{n j \delta}=X_{n j \delta}-E_{P} X_{n j \delta}$. By (a) and (b) of Proposition 2.4 (I) (or [24, Theorem 4.2]), $\lim _{n} E\left(\sum_{1}^{n} Y_{n j \delta}\right)=1$; moreover, $E\left(\sum_{1}^{n} Y_{n j \delta}\right)^{2} \leq\left(1+4 \sum_{1}^{\infty} \phi^{1 / 2}(j)\right) n E\left(X_{n 1}^{2} ;\left|X_{n 1}\right| \leq \delta\right)$ by an inequality of Ibragimov. Then, using that $\{x(n)\}$ is bounded and $b(n) \rightarrow \infty$ we obtain

$$
\frac{1}{2} \leq \operatorname{Mnb}(n)^{-2} E\left(f^{2}\left(a_{1}\right) ;\left|f\left(a_{1}\right)\right| \leq b(n)\right)
$$

if $n \geq n_{1}$ for some $M>0$ and $n_{1} \in \mathbf{N}^{*}$. Since $\lim _{n} n P\left(\left|f\left(a_{1}\right)\right|>b(n)\right)=0$ (use (a) of Proposition 2.4 (I) and that $x(n) / b(n) \rightarrow 0$ ) and $b(n+1) / b(n) \rightarrow 0$ we can conclude that $x^{2} P\left(\left|f\left(a_{1}\right)\right|>x\right)\left(E\left(f^{2}\left(a_{1}\right) ;\left|f\left(a_{1}\right)\right| \leq x\right)\right)^{-1} \rightarrow 0$ as $x \rightarrow \infty$, which says that $U^{\prime}$ is slowly varying.

2.10. Proposition. Let $f: \mathbf{N}^{*} \rightarrow \mathbf{R}$ and $\kappa_{1}, \kappa_{2}, \beta$ be such that $\kappa_{1} \geq 0$, $\kappa_{2} \geq 0, \kappa_{1}+\kappa_{2}>0, \beta \in(0,2)$. Denote by $\nu\left(\kappa_{1}, \kappa_{2}, \beta\right)$ the stable law $c_{1} \operatorname{Pois}\left(\mu\left(\kappa_{1}, \kappa_{2}, \beta\right)\right)$ with Lévy measure

$$
\mu\left(\kappa_{1}, \kappa_{2}, \beta\right)(d x)=\left\{I_{(-\infty, 0)}(x) \kappa_{2}|x|^{-1-\beta}+I_{(0, \infty)}(x) \kappa_{1} x^{-1-\beta}\right\} d x,
$$

i.e.,

$$
\begin{aligned}
\nu\left(\kappa_{1}, \kappa_{2}, \beta\right)^{\wedge}(y)=\exp \left\{\int_{-\infty}^{0}\left(e^{i x y}-1-i x y I_{[-1,0)}(x)\right) \kappa_{2}|x|^{-1-\beta} d x\right. \\
\left.+\int_{0}^{\infty}\left(e^{i x y}-1-i x y I_{(0,1]}(x)\right) \kappa_{1} x^{-1-\beta} d x\right\} .
\end{aligned}
$$

(a) Let $\{x(n)\} \subset \mathbf{R}$ and $\{b(n)\} \subset(0, \infty)$ with $b(n) \rightarrow \infty$. The following assertions are equivalent: (I) $\xi_{n}$ defined as in (2.6) satisfy

$$
\mathscr{L}_{\lambda}\left(\xi_{n}\right) \underset{w}{\rightarrow} Q_{\nu\left(\kappa_{1}, \kappa_{2}, \beta\right)}
$$

(II) $\mathscr{L}_{\lambda}\left(b(n)^{-1} \sum_{1}^{n}\left(f\left(a_{j}\right)-x(n)\right)\right) \underset{w}{\rightarrow} \nu\left(\kappa_{1}, \kappa_{2}, \beta\right)$ and $\left\{X_{n j}\right\}:=$ $\left\{b(n)^{-1}\left(f\left(a_{j}\right)-x(n)\right): 1 \leq j \leq n, n \geq 1\right\}$ satisfies $(*)$.

(b) The assertion

(A) There exist $\{x(n)\} \subset \mathbf{R}$ and $\{b(n)\} \subset(0, \infty)$ with $b(n) \rightarrow \infty$ such that (I) of (a) is satisfied,

holds if and only if

$$
R(x):=\sum_{k:|f(k)|>x} k^{-2} \text { is regularly varying with exponent }-\beta,
$$




$$
\lim _{x \rightarrow \infty} \frac{\sum_{k: f(k)>x} k^{-2}}{\sum_{k:|f(k)|>x} k^{-2}}=\frac{\kappa_{1}}{\kappa_{1}+\kappa_{2}}
$$

and

$$
\lim _{x \rightarrow \infty} \frac{\sum_{k: f(k)<-x} k^{-2}}{\sum_{k:|f(k)|>x} k^{-2}}=\frac{\kappa_{2}}{\kappa_{1}+\kappa_{2}} .
$$

If this is the case we can take $x(n)=E_{P}\left(f\left(a_{1}\right) ;\left|f\left(a_{1}\right)\right| \leq b(n)\right)$ and any $\{b(n)\}$ such that $\lim _{n} n b(n)^{-2} U(b(n))=\left(\kappa_{1}+\kappa_{2}\right)(1-\beta)^{-1}$ (with $U$ defined in (2.9)).

(c) If (I) holds for some $\{x(n)\},\{b(n)\}$, then (2.10) holds with $\lambda$ replaced by any $\rho \ll \lambda$.

Proof. (b) Assume that (II) holds. Proposition 2.4 implies that

$$
n \mathscr{L}_{P}\left(b(n)^{-1} f\left(a_{1}\right)\right)\left|B_{\tau}^{c} \underset{w}{\longrightarrow} \mu\left(\kappa_{1}, \kappa_{2}, \beta\right)\right| B_{\tau}^{c}
$$

for every $\tau>0$. To conclude the proof of the "only if" part see [2, pp. 81 and 84-85] and use (2.3). For the converse, apply Proposition 2.2 and argue as in [2, pp. 87-88].

We point out that if $x(n)=n x$ for some $x \in \mathbf{R}$ then the condition that $\left\{X_{n j}\right\}$ satisfies $(*)$ can be omitted in II of Propositions 2.9 and 2.10 [22, Theorem 2; 26, Remark 3.4.3.1].

Next we make some remarks about the validity of $(2.8)$ or (2.11) for certain positive functions $f$ of real argument.

Suppose $f:[1, \infty) \rightarrow(0, \infty)$ is bounded on finite intervals and $\lim _{x \rightarrow \infty} f(x)$ $=\infty$; then the following functions are well defined for $y \in[f(1), \infty)$

$$
\begin{gathered}
\bar{f}_{0}(y)=\inf \{x \geq 1: f(x) \geq y\}, \quad \bar{f}_{1}(y)=\inf \{x \geq 1: f(x)>y\}, \\
\bar{f}_{2}(y)=\sup \{x \geq 1: f(x) \leq y\} .
\end{gathered}
$$

We have $1 \leq \bar{f}_{0} \leq \bar{f}_{1} \leq \bar{f}_{2}$; each $\bar{f}_{i}$ is nondecreasing and $\lim _{x \rightarrow \infty} \bar{f}_{i}(x)=+\infty$ for such an $f$. We will say that $f \in \mathscr{F}$ if $f$ is Borel measurable, satisfies the preceding conditions and $\bar{f}_{1}(y) \sim \bar{f}_{2}(y)$ as $y \rightarrow \infty$.

2.11. Lemma. (i) If $f:[1, \infty) \rightarrow(0, \infty)$ is nondecreasing and $\lim _{x \rightarrow \infty} f(x)=$ $\infty$ then $f \in \mathscr{F}$.

(ii) If $f:[1, \infty) \rightarrow(0, \infty)$ is bounded on finite intervals and regularly varying with exponent $\alpha>0$ then $f \in \mathscr{F}$. Moreover $\bar{f}_{0}(y) \sim \bar{f}_{2}(y)$ as $y \rightarrow \infty$ and $\bar{f}_{i}$ is regularly varying with exponent $1 / \alpha \quad(i=0,1,2)$.

(iii) If $f \in \mathscr{F}$ and $\bar{f}_{1}$ is regularly varying with exponent $1 / \alpha$ for some $\alpha>0$ then $f$ is regularly varying with exponent $\alpha$.

Proof. (i) $\bar{f}_{1}=\bar{f}_{2}$ if $f$ is nondecreasing.

(ii) First we prove that $\bar{f}_{0} \sim \bar{f}_{2}$. We will show that for every $t>1$ we have $\bar{f}_{2}(y) \leq t \bar{f}_{0}(y)$ for all sufficiently large $y$ by using the Karamata representation: 
$f(x)=x^{\alpha} c(x) \exp \left(\int_{1}^{x} s^{-1} \varepsilon(s) d s\right), c$ and $\varepsilon$ being measurable functions with $\lim _{x \rightarrow \infty} c(x)=c>0, \lim _{s \rightarrow \infty} \varepsilon(s)=0$ (see [27]). Fix $t>1$ and take $r \in$ $(0,1)$ such that $r t^{\alpha / 2}>1$. There exists $y_{0}$ such that for every $y \geq y_{0}$ we have $\bar{f}_{0}(y)+1<t \bar{f}_{0}(y), r\left(t \bar{f}_{0}(y)\left(\bar{f}_{0}(y)+1\right)^{-1}\right)^{\alpha / 2}>1,|\varepsilon(s)| \leq \alpha / 2$ if $s \geq \bar{f}_{0}(y)$ and $c(x) / c\left(x^{\prime}\right) \geq r$ if $x, x^{\prime} \geq \bar{f}_{0}(y)$. Then if $y \geq y_{0}$ and $x>t \bar{f}_{0}(y)$, taking $x^{\prime}$ such that $\bar{f}_{0}(y) \leq x^{\prime}<\bar{f}_{0}(y)+1$ and $f\left(x^{\prime}\right) \geq y$, we have $f(x) / y \geq$ $r\left(x / x^{\prime}\right)^{\alpha / 2}>1$; this implies that $\bar{f}_{2}(y) \leq t \bar{f}_{0}(y)$ if $y \geq y_{0}$.

Now fix $t>0$. Given $r>1$, by hypothesis we have

$$
\lim _{y \rightarrow \infty} f\left(r^{-1} t^{-1 / \alpha} \bar{f}_{1}(t y)\right) / f\left(\bar{f}_{1}(t y)-1\right)=r^{-\alpha} t^{-1}
$$

which implies that, for all sufficiently large $y$,

$$
f\left(r^{-1} t^{-1 / \alpha} \bar{f}_{1}(t y)\right) \leq t^{-1} f\left(\bar{f}_{1}(t y)-1\right) \leq y
$$

by the definition of $\bar{f}_{1}$ and $r^{-1} t^{-1 / \alpha} \bar{f}_{1}(t y) \leq \bar{f}_{2}(y)$ by the definition of $\bar{f}_{2}$. Then $\lim \sup _{y \rightarrow \infty} \bar{f}_{1}(t y) / \bar{f}_{2}(y) \leq t^{1 / \alpha}$. By a similar argument we can deduce from the fact that

$$
\lim _{y \rightarrow \infty} f\left(r t^{1 / \alpha} \bar{f}_{1}(y)\right) / f\left(\bar{f}_{1}(y)-1\right)=r^{\alpha} t
$$

for each $r \in(0,1)$ that $\liminf _{y \rightarrow \infty} \bar{f}_{2}(t y) / \bar{f}_{1}(y) \geq t^{1 / \alpha}$. This implies that $\bar{f}_{i}$ varies regularly with exponent $1 / \alpha$ because $\bar{f}_{0}, \bar{f}_{1}, \bar{f}_{2}$ are asymptotically equivalent.

(iii) Take $t>0$. For any $r>1$, the hypotheses give that

$$
\lim _{x \rightarrow \infty} \bar{f}_{1}\left(r t^{\alpha} f(x)\right) / \bar{f}_{2}(f(x))=r^{1 / \alpha} t
$$

which implies that, for all sufficiently large $x, \bar{f}_{1}\left(r t^{\alpha} f(x)\right)>t \bar{f}_{2}(f(x)) \geq t x$ by the definition of $\bar{f}_{2}$ and $f(t x) \leq r t^{\alpha} f(x)$ by the definition of $\bar{f}_{1}$. Then $\limsup _{x \rightarrow \infty} f(t x) / f(x) \leq t^{\prime *}$. On the other hand

$$
\lim _{x \rightarrow \infty} \bar{f}_{1}\left(r^{-1} t^{-\alpha} f(t x)\right) / \bar{f}_{2}(f(t x))=r^{-1 / \alpha} t^{-1}
$$

for each $r \in(0,1)$ and an analogous argument shows that

$$
\liminf _{x \rightarrow \infty} f(t x) / f(x) \geq t^{\alpha} .
$$

2.12. Corollary. (a) Let $f \in \mathscr{F}$. Then assertion (A) of Proposition 2.9 holds if and only if

$$
\lim _{x \rightarrow \infty} \frac{x^{-1} f^{2}(x)}{\sum_{k: k \leq x} f^{2}(k) k^{-2}}=0 .
$$

Moreover, in this case $U$ (defined in (2.9)) is asymptotically equivalent to

$$
\tilde{U}(x)=(\log 2)^{-1} \sum_{k: k \leq \bar{f}_{2}(x)} f^{2}(k) k^{-2} ;
$$

here $\bar{f}_{2}$ can be replaced by $\bar{f}_{1}$. 
(b) If $f:[1, \infty) \rightarrow(0, \infty)$ is regularly varying with exponent $\alpha=1 / 2$ and bounded on finite intervals then (A) of Proposition 2.9 holds.

Proof. (a) Assume that $f$ satisfies (2.12). We claim

$$
\sum_{k<\bar{f}_{1}(y)} f^{2}(k) k^{-2} \sim \sum_{k \leq \bar{f}_{2}(y)+1} f^{2}(k) k^{-2} \text { as } y \rightarrow \infty .
$$

Write

$$
g(y)=\left(\sum_{k \leq \bar{f}_{2}(y)+1} f^{2}(k) k^{-2}\right) /\left(\sum_{k<\bar{f}_{1}(y)} f^{2}(k) k^{-2}\right) .
$$

Let $\varepsilon \in(0,1 / 2)$. There exists $y_{0}$ such that if $y \geq y_{0}$ then $f^{2}(z) z^{-2} \leq$ $\varepsilon z^{-1} \sum_{h \leq z} f^{2}(h) h^{-2}$ for $z \geq \bar{f}_{1}(y)$ and $\log \left(\left(\bar{f}_{2}(y)+1\right) /\left(\bar{f}_{1}(y)-1\right)\right) \leq 2$. Therefore if $y \geq y_{0}$

$$
\sum_{\bar{f}_{1}(y) \leq k \leq \bar{f}_{2}(y)+1} f^{2}(k) k^{-2} \leq 2 \varepsilon \sum_{h \leq \bar{f}_{2}(y)+1} f^{2}(h) h^{-2}
$$

which implies $1 \leq g(y) \leq 1+2 \varepsilon g(y)$, that is $1 \leq g(y) \leq(1-2 \varepsilon)^{-1}$. This proves (2.13).

By the definitions of $\bar{f}_{1}$ and $\bar{f}_{2}$ we have

$$
y^{2} \sum_{k: f(k)>y} k^{-2} \leq y^{2} \sum_{k \geq \bar{f}_{1}(y)} k^{-2} \leq \frac{f^{2}\left(\bar{f}_{2}(y)+1\right)}{\bar{f}_{1}(y)-1}
$$

and

$$
\sum_{k: f(k) \leq y} f^{2}(k) k^{-2} \geq \sum_{k<\bar{f}_{1}(y)} f^{2}(k) k^{-2} .
$$

Then using that $\bar{f}_{1} \sim \bar{f}_{2},(2.13)$ and (2.12) we obtain (2.8). That $U \sim \tilde{U}$ follows from (2.13) and the inequalities

$$
\sum_{k<\bar{f}_{1}(y)} f^{2}(k) k^{-2} \leq \sum_{k: f(k) \leq y} f^{2}(k) k^{-2} \leq \sum_{k \leq \bar{f}_{2}(y)} f^{2}(k) k^{-2} .
$$

Now suppose that $f$ satisfies (2.8). Write $u(x)$ for the quotient in (2.12). First observe that

$$
\sum_{k: f(k) \leq f(x)-1} f^{2}(k) k^{-2} \leq \sum_{k \leq x} f^{2}(k) k^{-2}+2 \frac{f^{2}(x)}{x}
$$

$(x \geq 1)$ and that for some constant $C$

$$
\frac{1}{x} \leq C \sum_{k: f(k)>f(x)-1} k^{-2}
$$

for all sufficiently large $x$ (by the definitions of $\bar{f}_{1}$ and $\bar{f}_{2}$ we have $x \geq$ $\bar{f}_{1}(f(x)-1)$ and

$$
\left(\bar{f}_{2}(f(x)-1)+1\right)^{-1} \leq \sum_{k>\bar{f}_{2}(f(x)-1)} k^{-2} \leq \sum_{k: f(k)>f(x)-1} k^{-2}
$$


moreover, $\bar{f}_{1} \sim \bar{f}_{2}$ and $f(x) \rightarrow \infty$ as $\left.x \rightarrow \infty\right)$. Therefore we have for such $x$ 's

$$
\begin{aligned}
u(x) & \leq C \frac{f^{2}(x) \sum_{k: f(k)>f(x)-1} k^{-2}}{\sum_{k \leq x} f^{2}(k) k^{-2}} \\
& \leq C(1+2 u(x)) \frac{f^{2}(x) \sum_{k: f(k)>f(x)-1} k^{-2}}{\sum_{k: f(k) \leq f(x)-1} f^{2}(k) k^{-2}} .
\end{aligned}
$$

Since $f(x) \rightarrow \infty$ as $x \rightarrow \infty,(2.8)$ implies that for any $\varepsilon \in\left(0,(2 C)^{-1}\right)$ we have $u(x) \leq C(1+2 u(x)) \varepsilon$ and hence $u(x) \leq \varepsilon C(1-2 \varepsilon C)^{-1}$ for all sufficiently large $x$. This implies (2.12).

(b) Use (a), Lemma 2.11(ii) and [2, Chapter 2, Lemma 6.15].

2.13. Corollary. (a) Let $\kappa_{1}, \kappa_{2}, \beta$ be as in Proposition 2.10 with $\beta=1 / \alpha$, $\alpha>1 / 2$. Let $f \in \mathscr{F}$. Then (A) of Proposition 2.10 holds if and only if $f$ is regularly varying with exponent $\alpha$.

(b) Assume $f:[1, \infty) \rightarrow(0, \infty)$ is regularly varying with exponent $\alpha>1 / 2$, bounded on finite intervals. Let

$$
\nu_{\alpha}= \begin{cases}\delta_{((\alpha-1) \log 2)^{-1}} * \nu\left(\frac{1}{\alpha \log 2}, 0, \frac{1}{\alpha}\right) & \text { if } \alpha \neq 1, \\ \nu\left(\frac{1}{\log 2}, 0,1\right) & \text { if } \alpha=1,\end{cases}
$$

$\left(\nu(., .,\right.$.$) defined as in Proposition 2.10) and define \xi_{n}$ by

$$
\xi_{n}(t)=f(n)^{-1} \sum_{1 \leq j \leq[n t]} f\left(a_{j}\right) \quad \text { if } \alpha>1,
$$

$$
\xi_{n}(t)=f(n)^{-1} \sum_{1 \leq j \leq[n t]}\left\{f\left(a_{j}\right)-E_{P}\left(f\left(a_{1}\right) ; f\left(a_{1}\right) \leq f(n)\right)\right\} \quad \text { if } \alpha=1
$$

$$
\xi_{n}(t)=f(n)^{-1} \sum_{1 \leq j \leq[n t]}\left\{f\left(a_{j}\right)-E_{P} f\left(a_{1}\right)\right\} \quad \text { if } \frac{1}{2}<\alpha<1 .
$$

Then for any $\rho \ll \lambda, \mathscr{L}_{\rho}\left(\xi_{n}\right) \rightarrow{ }_{w} Q_{\nu_{\alpha}}$.

Proof. (a) Since $f \in \mathscr{F}$, by Lemma 2.11 it is sufficient to show

$$
\sum_{k: f(k)>x} k^{-2} \sim \frac{1}{\bar{f}_{1}(x)} \text { as } x \rightarrow \infty .
$$

By the definitions of $\bar{f}_{1}$ and $\bar{f}_{2}$

moreover

$$
1 \leq \frac{\sum_{k: f(k)>x} k^{-2}}{\sum_{k>\bar{f}_{2}(x)} k^{-2}} \leq 1+\frac{\sum_{k: \bar{f}_{1}(x) \leq k \leq \bar{f}_{2}(x)} k^{-2}}{\sum_{k>\bar{f}_{2}(x)} k^{-2}}=1+v(x) \text { (say); }
$$

$$
\sum_{k: \bar{f}_{1}(x) \leq k \leq \bar{f}_{2}(x)} k^{-2} \leq\left(\bar{f}_{1}(x)-1\right)^{-1}-\left(\bar{f}_{2}(x)\right)^{-1}
$$


and

$$
\sum_{k>\bar{f}_{2}(x)} k^{-2} \geq\left(\bar{f}_{2}(x)+1\right)^{-1} .
$$

Then $\lim _{x \rightarrow \infty} v(x)=0$ and (2.18) holds since

$$
\sum_{k>\bar{f}_{2}(x)} k^{-2} \sim\left(\bar{f}_{2}(x)\right)^{-1} \sim\left(\bar{f}_{1}(x)\right)^{-1} \text { as } x \rightarrow \infty .
$$

(b) From [2, Chapter 2, Lemma 6.15] we obtain

$$
\lim _{x \rightarrow \infty} \frac{\sum_{k \leq x} f^{2}(k) k^{-2}}{x^{-1} f^{2}(x)}=\frac{1}{2 \alpha-1} .
$$

On the other hand

$$
1 \leq \frac{\sum_{k: f(k) \leq x} f^{2}(k) k^{-2}}{\sum_{k<\bar{f}_{1}(x)} f^{2}(k) k^{-2}} \leq \frac{\sum_{k \leq \bar{f}_{2}(x)} f^{2}(k) k^{-2}}{\sum_{k<\bar{f}_{1}(x)} f^{2}(k) k^{-2}}
$$

which by (2.19) goes to one as $x \rightarrow \infty$ because $\bar{f}_{1} \sim \bar{f}_{2}$ and $f$ is regularly varying. Then by (2.9) and (2.19)

$$
\frac{n}{f^{2}(n)} U(f(n)) \sim \frac{1}{\log 2} \frac{n}{f^{2}(n)} \sum_{k<\bar{f}_{1}(f(n))} f^{2}(k) k^{-2} \sim \frac{1}{(2 \alpha-1) \log 2}
$$

because $f$ is regularly varying and $\bar{f}_{1}(f(n)) \sim n$ as $n \rightarrow \infty$ (observe that

$$
\bar{f}_{1}(f(n))\left(\bar{f}_{2}(f(n))\right)^{-1} \leq \bar{f}_{1}(f(n)) n^{-1} \leq \bar{f}_{1}(f(n)) \bar{f}_{1}(f(n)-1)
$$

and $\bar{f}_{1}$ is regularly varying). Therefore we can take $b(n)=f(n), \kappa_{1}=$ $(\alpha \log 2)^{-1}, \kappa_{2}=0$ in $(2.10)$.

This result implies that if $f$ satisfies the assumptions in (b) with $\alpha \in(1 / 2,1)$ then

$$
\mathscr{L}_{\rho}\left(\frac{1}{n} \operatorname{card}\left\{k \leq n: \frac{1}{k} \sum_{1}^{k} f\left(a_{j}\right)>E_{p} f\left(a_{1}\right)\right\}\right)
$$

converges to the law given in [1, Theorem 5.2] (observe that for such an $\alpha$, $\nu_{r}$ is strictly stable and satisfies $0<\nu_{r r}((0, \infty))<1$ - use [9, Chapter IV, $\S 1$, Theorem 7]). 


\subsection{Examples.}

2.14.1. Let $f(x)=x^{1 / 2}$ and take $b(n)=(n \log n / \log 2)^{1 / 2}, x(n)=E_{P}\left(a_{1}^{1 / 2}\right)$ in (2.6). Then (2.7) holds with $\lambda$ replaced by any $\rho \ll \lambda$ (observe that $\widetilde{U}(x) \sim$ $\left.(\log 2)^{-1} 2 \log x\right)$.

2.14.2. If $\xi_{n}$ is defined by

$$
\xi_{n}(t)=\frac{1}{n} \sum_{1 \leq j \leq[n t]}\left\{a_{j}-\frac{\log n}{\log 2}\right\} \quad(t \in[0,1]),
$$

then for any $\rho \ll \lambda, \mathscr{L}_{\rho}\left(\xi_{n}\right) \underset{w}{\rightarrow} Q_{\nu^{\prime}}$, where $\nu^{\prime}=\delta_{x} * \nu(1 / \log 2,0,1)$, i.e.,

$$
\hat{\nu}^{\prime}(y)=\exp \left\{i x y+\int_{0}^{\infty}\left(e^{i x y}-1-i x y I_{(0,1]}(x)\right) \frac{1}{\log 2} x^{-2} d x\right\}
$$

with

$$
x=\lim _{n} \frac{1}{\log 2}\left(\sum_{k=1}^{n} k \log \left(1+\frac{1}{k(k+2)}\right)-\log n\right) .
$$

As a consequence, if $\mathscr{L}(\xi)=Q_{\nu^{\prime}}$,

$$
\mathscr{L}_{\rho}\left(\frac{1}{n} \operatorname{card}\left\{k \leq n: \frac{1}{k} \sum_{1}^{k} a_{j}>\frac{\log n}{\log 2}\right\}\right) \underset{w}{ } \mathscr{L}(\lambda\{t \in[0,1]: \xi(t)>0\})=\sigma
$$

(say). We do not know an explicit expression for $\sigma$ (observe that $\nu^{\prime}$ is not strictly stable; on the other hand, [9, Chapter IV, $§ 1$, Theorem 7] shows that $\left.\sigma \neq \delta_{0}, \sigma \neq \delta_{1}\right)$.

2.14.3. Let $\alpha \geq 1 / 2$ and $c>0$ with $c \alpha>\left(\alpha^{2}+1\right)^{1 / 2}$; then $f(x)=$ $x^{\alpha}(c+\sin (\log x))$ belongs to $\mathscr{F}$ and is not regularly varying. Hence if $\alpha>1 / 2$, $f$ does not verify $(\mathrm{A})$ of Proposition 2.10 (this is related to [18, footnote on p. 199]). If $\alpha=1 / 2, f$ satisfies (2.12) and (A) of Proposition 2.9 holds with $x(n)=E_{P} f\left(a_{1}\right), b(n)=\left(\left(c^{2}-\frac{1}{2}\right)(\log 2)^{-1} n \log n\right)^{1 / 2}$ (we have $\tilde{U}(x) \sim$ $\left(c^{2}-\frac{1}{2}\right)(\log 2)^{-1} \log \left(f^{-1}(x)\right)$; writing $h(x)=\log \left(f^{-1}(x)\right)$ we obtain $h(x)+$ $2 \log (c+\sin h(x))=2 \log x$ which implies $h(x) \sim 2 \log x$. Then $\widetilde{U}(x) \sim$ $\left.\left(2 c^{2}-1\right)(\log 2)^{-1} \log x\right)$.

2.15. Remarks. Lévy [18] proves the convergence of $\mathscr{L}_{\rho}\left(\xi_{n}(1)\right)$ of Corollary 2.13 for nondecreasing regularly varying functions (see also [19, Chapitre IX]); the case $f(x)=x$ (which improves a result of Khintchine [14, p. 377]) was also given by Doeblin [7] (for $\rho=\lambda$ ) and by Philipp [23] (using [24]). The assertion that $\mathscr{L}_{\lambda}\left(\xi_{n}(1)\right)$ of Example 2.14.1 converges to the normal law is stated in [19, Chapitre IX] without indicating the norming constants.

\section{COMPARISON WITH OTHER SUMS}

Throughout this section, $\left\{\eta_{n i}: 1 \leq j \leq n, n \geq 1\right\}$ denotes a double array of measurable real functions on $(\Omega, \mathscr{B})$. 
Define

$$
\mathscr{M}_{j l}= \begin{cases}\sigma\left(a_{j-l}, \ldots, a_{j+l}\right) & \text { if } j-l \geq 1, \\ \sigma\left(a_{1}, \ldots, a_{j+l}\right) & \text { if } j-l<1 .\end{cases}
$$

For the proof of the following inequality see [5, pp. 188-190].

3.1. Lemma. Assume $E_{P} \eta_{n j}^{2}<\infty$ for all $n, j$. If

$$
\mu_{n}(p):=\sum_{l=p}^{\infty} \max _{1 \leq j \leq n} E_{P}^{1 / 2}\left(\eta_{n j}-\eta_{n j l}\right)^{2}
$$

where $\eta_{n j l}:=E_{P}\left(\eta_{n j} \mid \mathscr{M}_{j l}\right)$, and

$$
\beta_{n}(p, \varepsilon):=\max _{0 \leq k \leq n-2 p} P\left(\sum_{j=k+1}^{k+2 p}\left|\eta_{n j}\right|>\varepsilon\right)
$$

then for any $\varepsilon>0, n \geq 1,1 \leq p \leq n / 2$ we have

$$
\begin{aligned}
P\left(\max _{1 \leq i \leq n}\left|\sum_{j=1}^{i} \eta_{n j}\right|>6 \varepsilon\right) \leq & \phi(2 p)+4(2 / \varepsilon)^{2} n \mu_{n}^{2}(p)+4 n \beta_{n}(p, \varepsilon / 2) \\
& +2 \max _{1 \leq i \leq n} P\left(\left|\sum_{j=i}^{n} \eta_{n j}\right|>\varepsilon\right) .
\end{aligned}
$$

\subsection{Lemma. Assume}

(1) $E_{P} \eta_{n j}^{2}<\infty$ for all $n, j$.

(2) $\lim _{p} \sup _{n} n \mu_{n}^{2}(p)=0 \quad\left(\mu_{n}\right.$ defined in (3.2)).

(3) $\lim _{n} n \max _{1 \leq j \leq n} P\left(\left|\eta_{n j}\right|>\varepsilon\right)=0$ for each $\varepsilon>0$.

(4) $\lim _{n} \max _{1 \leq i \leq n} P\left(\left|\sum_{j=i}^{n} \eta_{n j}\right|>\varepsilon\right)=0$ for each $\varepsilon>0$.

Then $\max _{1 \leq i \leq n}\left|\sum_{j=1}^{i} \eta_{n j}\right| \rightarrow 0$ in measure.

Proof. Let $\varepsilon>0$. By Lemma 3.1 it suffices to find $p_{n} \rightarrow \infty, p_{n} \leq n / 2$ such that $\lim _{n} n \beta_{n}\left(p_{n}, \varepsilon\right)=0$. This can be obtained from (3), noting that $n \beta_{n}(p, \varepsilon) \leq 2 p n \max _{j \leq n} P\left(\left|\eta_{n j}\right|>\varepsilon /(2 p)\right)$ for each $p$ (this is an argument in $[5$, p. 175]).

\subsection{Proposition. Assume}

(1) $E_{P} \eta_{n j}^{2}<\infty, E_{P} \eta_{n j}=0$ for all $n, j$.

(2) $\lim _{n} n \max _{1 \leq j \leq n} E_{P} \eta_{n j}^{2}=0$.

(3) $\lim _{p} \sup _{n} n \mu_{n}^{2}(p)=0 \quad\left(\mu_{n}\right.$ defined in (3.2)).

Then $\max _{1 \leq i \leq n}\left|\sum_{j=1}^{i} \eta_{n j}\right| \rightarrow 0$ in measure.

Proof. In order to verify that (4) of Lemma 3.2 holds it is sufficient to show that

$$
\lim _{n} \max _{1 \leq i \leq n} E_{P}\left(\sum_{j=i}^{n} \eta_{n j}\right)^{2}=0
$$


Write $M_{n}=\max _{j \leq n} E_{P} \eta_{n j}^{2}$ and $\nu_{n j}(l)=E_{P}\left(\eta_{n j}-\eta_{n j l}\right)^{2}$. Let $1 \leq j<k \leq n$ with $k-j \geq 3$. If $l=[(k-j) / 3]$ arguing as in [11, p. 369] (or [5, p. 185]) by conditioning with respect to $\mathscr{M}_{j l}$ and $\mathscr{M}_{k l}$ we obtain

$$
\begin{aligned}
\left|E_{P} \eta_{n j} \eta_{n k}\right| \leq & 2 \phi^{1 / 2}\left(\left[\frac{k-j}{3}\right]\right) M_{n}+2\left(M_{n} \max _{i \leq n} \nu_{n i}\left(\left[\frac{k-j}{3}\right]\right)\right)^{1 / 2} \\
& +\max _{i \leq n} \nu_{n i}\left(\left[\frac{k-j}{3}\right]\right) .
\end{aligned}
$$

Therefore, writing $K_{n 0}=K_{n 1}=K_{n 2}=M_{n}$ and

$$
K_{n h}=2 \phi^{1 / 2}\left(\left[\frac{h}{3}\right]\right) M_{n}+2\left(M_{n} \max _{i \leq n} \nu_{n i}\left(\left[\frac{h}{3}\right]\right)\right)^{1 / 2}+\max _{i \leq n} \nu_{n i}\left(\left[\frac{h}{3}\right]\right)
$$

for $h \geq 3$, we get $\left|E_{P} \eta_{n j} \eta_{n k}\right| \leq K_{n, k-j}$ if $1 \leq j \leq k \leq n$. Then if $1 \leq i \leq n$

$$
\begin{aligned}
E_{P}\left(\sum_{j=i}^{n} \eta_{n j}\right)^{2} \leq & n\left\{K_{n 0}+2 \sum_{h=1}^{\infty} K_{n h}\right\} \\
= & 5 n M_{n}+4 n M_{n} \sum_{h=3}^{\infty} \phi^{1 / 2}\left(\left[\frac{h}{3}\right]\right) \\
& +4\left(n M_{n}\right)^{1 / 2} n^{1 / 2} \sum_{h=3}^{\infty} \max _{i \leq n} \nu_{n i}^{1 / 2}\left(\left[\frac{h}{3}\right]\right) \\
& +2 n \sum_{h=3}^{\infty} \max _{i \leq n} \nu_{n i}\left(\left[\frac{h}{3}\right]\right) .
\end{aligned}
$$

From this one can obtain (3.3).

We will use only the following.

3.4. Corollary. Let $\left\{\eta_{j}: j \geq 1\right\}$ be a sequence of measurable real functions on $(\Omega, \mathscr{B})$ and $\{b(n): n \geq 1\} \subset(0, \infty)$. Assume

(1) $\sup _{j} E_{P} \eta_{j}^{2}<\infty$ and $E_{P} \eta_{j}=0$ for every $j \geq 1$.

(2) $\lim _{n} n b(n)^{-2}=0$.

(3) $\sum_{l=1}^{\infty} \sup _{j} E_{P}^{1 / 2}\left(\eta_{j}-E_{P}\left(\eta_{j} \mid \mathscr{M}_{j l}\right)\right)^{2}<\infty$.

Then $\max _{1 \leq i \leq n}\left|b(n)^{-1} \sum_{j=1}^{i} \eta_{j}\right| \rightarrow 0$ in measure.

Proof. Write $\eta_{n j}=b(n)^{-1} \eta_{j}$ and observe that

$$
\sup _{n} n \mu_{n}^{2}(p) \leq\left(\sup _{n} n b(n)^{-2}\right)\left(\sum_{l=p}^{\infty} \sup _{j} E_{P}^{1 / 2}\left(\eta_{j}-E_{P}\left(\eta_{j} \mid \mathscr{M}_{j l}\right)\right)^{2}\right)^{2} .
$$




\section{Complete Quotients AND The SEQuence $\left\{u_{j}\right\}$}

Following Doeblin [7, p. 365] we write for $\omega \in \Omega, j \geq 1$,

$$
\frac{1}{u_{j}(\omega)}=\left|\omega-\frac{p_{j-1}(\omega)}{q_{j-1}(\omega)}\right| q_{j-1}^{2}(\omega) \text {. }
$$

Then $u_{1}(\omega)=x_{1}(\omega)$ and $u_{j}(\omega)=x_{j}(\omega)+\left(y_{j-1}(\omega)\right)^{-1}$ if $j \geq 2$.

We will try to extend some results of $\S 2$ to $\left\{x_{j}\right\}$ and $\left\{u_{j}\right\}$. In our first statements, if $\xi$ is a random element defined in terms of the $a_{j}$ 's, $\tilde{\xi}$ denotes that one obtained by replacing the $a_{j}$ 's by the $x_{j}$ 's; $\tilde{\tilde{\xi}}$ is similarly defined when considering the $u_{j}$ 's. For instance, if $\xi_{n}$ is as in Example 2.6.2(c), $\tilde{\xi}_{n}(t)=$ $\operatorname{card}\left\{j \leq[n t]: u_{j}>\theta n\right\}$.

\subsection{Examples.}

4.1.1. Let $\theta, \alpha$ and $\xi_{n}$ be as in Example 2.6.2. Then the conclusion there remains valid for $\tilde{\xi}_{n}$ and $\tilde{\xi}_{n}$.

Proof. We have $\sup _{t \in[0,1]}\left|\tilde{\tilde{\xi}}_{n}(t)-\xi_{n}(t)\right| \leq \sum_{j=1}^{n}\left|\eta_{n j}\right|$ where

$$
\eta_{n j}=n^{-\alpha}\left(u_{j}^{\alpha} I_{\left\{u_{j}>\theta n\right\}}-a_{j}^{\alpha} I_{\left\{a_{j}>\theta n\right\}}\right) \text {. }
$$

Write

$$
\begin{aligned}
\sum_{j=1}^{n}\left|\eta_{n j}\right| \leq & n^{-\alpha} \sum_{j=1}^{n} u_{j}^{\alpha} I_{\left\{u_{j}>\theta n, a_{j} \leq \theta n\right\}} \\
& +n^{-\alpha} \sum_{j=1}^{n}\left|u_{j}^{\alpha}-a_{j}^{\alpha}\right| I_{\left\{a_{j}>\theta n\right\}}=X_{n}+Y_{n} \quad \text { (say). }
\end{aligned}
$$

Note that $P\left(X_{n}>0\right) \leq n P\left(\theta n-2<a_{1} \leq \theta n\right) \rightarrow 0$ (observe that $a_{j} \leq u_{j} \leq$ $\left.a_{j}+2\right)$ and, since

$$
Y_{n} \leq c_{\alpha x} n^{-1} n^{-(\alpha-1)} \sum_{j \leq n} a_{j}^{\alpha-1} I_{\left\{a_{j}>\theta n\right\}}
$$

with $c_{\alpha}=2 \alpha 3^{\alpha-1}$ if $\alpha \geq 1,=2|\alpha|$ if $\alpha<1,2.6 .2$ shows that $Y_{n} \rightarrow 0$ in measure. The proof for $\tilde{\xi}_{n}$ is similar.

4.1.2. The statement of Example 2.6 .3 is true if we put everywhere ${ }^{\sim}\left(\right.$ or $\left.^{z}\right)$ over the random elements there.

Proof. (Case $\tilde{\xi}_{n}$ ) Let $\eta_{n j}=f_{n}\left(u_{j}\right)-f_{n}\left(a_{j}\right), f_{n}$ being defined as in Example 2.6.3; it is sufficient to show that $\sum_{j=1}^{n}\left\|\eta_{n j}\right\| \rightarrow_{P} 0$. We have

$$
\left\|\eta_{n j}\right\|^{2} \leq 2 \sum_{r=1}^{\infty}\left(I_{A_{n j r}}+I_{B_{n j r}}\right)
$$


where

$$
\begin{aligned}
& A_{n j r}=\left\{\theta_{r} n<u_{j} \leq \theta_{r+1} n, a_{j} \leq \theta_{r} n\right\}, \\
& B_{n j r}=\left\{\theta_{r} n<a_{j} \leq \theta_{r+1} n, a_{j}>\theta_{r+1} n-2\right\} .
\end{aligned}
$$

Then

$$
\sum_{j=1}^{n} P\left(\left\|\eta_{n j}\right\|>0\right) \leq \sum_{j=1}^{n} \sum_{r=1}^{\infty} P\left(A_{n j r}\right)+\sum_{j=1}^{n} \sum_{r=1}^{\infty} P\left(B_{n j r}\right)=\alpha_{n}+\beta_{n} \text { (say). }
$$

Writing $\alpha_{n r}=\sum_{j=1}^{n} P\left(A_{n j r}\right)$ we have $\alpha_{n}=\sum_{r=1}^{\infty} \alpha_{n r}$. Note that $\alpha_{n r} \leq$ $n P\left(\theta_{r} n-2<a_{1} \leq \theta_{r} n\right) \rightarrow 0$ as $n \rightarrow \infty$ for each $r$. Moreover, given $r_{0} \geq 1$,

$$
\sum_{r \geq r_{0}} \alpha_{n r} \leq \sum_{j=1}^{n} P\left(u_{j}>\theta_{r_{0}} n\right) \leq n P\left(a_{1}>\theta_{r_{0}} n-2\right)
$$

which tends to $\left(\theta_{r_{0}} \log 2\right)^{-1}$ as $n \rightarrow \infty$. Then for every $r_{0} \geq 1, \lim \sup _{n} \alpha_{n} \leq$ $\left(\theta_{r_{0}} \log 2\right)^{-1}$; hence $\lim _{n} \alpha_{n}=0$. Analogously $\lim _{n} \beta_{n}=0$.

Now we turn to sums of the form $\sum_{1}^{n} f\left(x_{j}\right), \sum_{1}^{n} f\left(u_{j}\right)$. From Corollary 2.13 we obtain

4.2. Corollary. Assume $f$ is as in Corollary 2.13(b) with $\alpha>1$. If $\xi_{n}$ is defined by (2.15) then $\tilde{\xi}_{n}$ and $\tilde{\xi}_{n}$ satisfy the conclusion there.

Proof. (Case $\tilde{\xi}_{n}$ ) We will show that $X_{n}:=f(n)^{-1} \sum_{j=1}^{n}\left|f\left(u_{j}\right)-f\left(a_{j}\right)\right| \rightarrow_{P} 0$. Write $f(x)=x^{(x} L(x), L$ being slowly varying. We have

$$
\begin{aligned}
X_{n} & \leq f(n)^{-1} \sum_{j=1}^{n} u_{j}^{\alpha}\left|L\left(u_{j}\right)-L\left(a_{j}\right)\right|+f(n)^{-1} \sum_{j=1}^{n}\left(u_{j}^{\alpha}-a_{j}^{\alpha}\right) L\left(a_{j}\right) \\
& =X_{n 1}+X_{n 2} \text { (say). }
\end{aligned}
$$

Since $u_{j}^{\alpha}-a_{j}^{\alpha} \leq \alpha 3^{\alpha-1} a_{j}^{\alpha-1}$, then $X_{n 2} \rightarrow_{P} 0$ will follow if we show that $M_{n 2}:=f(n)^{-1} \sum_{j=1}^{n} a_{j}^{-1} f\left(a_{j}\right) \rightarrow_{p} 0$. Observe that if $K \geq 1$

$$
M_{n 2} \leq\left(\max _{i \in\{1, \ldots, K\}} \frac{f(i)}{i}\right) \frac{n}{f(n)}+\frac{1}{K} \frac{1}{f(n)} \sum_{j=1}^{n} f\left(a_{j}\right)
$$

(write $1=I_{\left\{a_{j} \leq K\right\}}+I_{\left\{a_{j}>K\right\}}$ in each term). Then given $\varepsilon>0$ we conclude by Corollary 2.13 that for every $K \geq 1$

$$
\begin{aligned}
\varlimsup_{n} P\left(M_{n 2}>\varepsilon\right) & \leq \varlimsup_{n} P\left(\xi_{n}(1)>K(\varepsilon / 2)\right) \\
& \leq \nu_{\alpha}(\{x: x \geq K(\varepsilon / 2)\})
\end{aligned}
$$

which goes to zero as $K \rightarrow \infty$. Hence $M_{n 2} \rightarrow_{P} 0$.

On the other hand, for each $K \geq 1$

$$
X_{n 1} \leq\left(1+3^{\alpha}\right) C_{K} \frac{n}{f(n)}+\frac{3^{\alpha}}{f(n)} \sum_{j=1}^{n} f\left(a_{j}\right)\left|\frac{L\left(u_{j}\right)}{L\left(a_{j}\right)}-1\right| I_{\left\{a_{j}>K\right\}}
$$


where $C_{K}=\sup _{1 \leq x \leq K+2} f(x)$ (finite by hypothesis). Let $\varepsilon>0$. Given $\eta>0$ take $K \geq 1$ such that $\sup _{0 \leq s \leq 2}\left|L(x)^{-1} L(x+s)-1\right| \leq \eta$ if $x>K$ (possible by the Karamata representation of $L)$; then

$$
\begin{aligned}
\varlimsup_{n} P\left(X_{n 1}>\varepsilon\right) & \leq \varlimsup_{n} P\left(\xi_{n}(1)>\left(\eta 3^{\alpha}\right)^{-1}(\varepsilon / 2)\right) \\
& \leq \nu_{\alpha}\left(\left\{x: x \geq\left(\eta 3^{\alpha}\right)^{-1}(\varepsilon / 2)\right\}\right)
\end{aligned}
$$

which tends to zero as $\eta \rightarrow 0$. Then $X_{n 1} \rightarrow_{P} 0$.

4.3. Lemma. Assume $f:[1, \infty) \rightarrow(0, \infty)$ is Borel measurable and satisfies there exist $r>0$ and $M: \mathbf{N}^{*} \rightarrow[0, \infty)$ with $E_{P} M^{2}\left(a_{1}\right)<\infty$ such that for every $k \in \mathbf{N}^{*},|f(x)-f(y)| \leq M(k)|x-y|^{r}$ if $x, y \in[k, k+2]$.

Let $\{b(n)\} \subset(0, \infty)$ such that $\lim _{n} n b(n)^{-2}=0$. Then if $\eta_{j}=f\left(u_{j}\right)-f\left(a_{j}\right)-$ $E_{P}\left(f\left(u_{j}\right)-f\left(a_{j}\right)\right)(j \geq 1)$ we have $\max _{1 \leq i \leq n}\left|b(n)^{-1} \sum_{j=1}^{i} \eta_{j}\right| \rightarrow 0$ in measure. The same result holds if we replace everywhere $u_{j}$ by $x_{j}$ in the definition of $\eta_{j}$. Proof. (Case $\left.\left\{u_{j}\right\}\right)$ Since $\left|f\left(u_{j}\right)-f\left(a_{j}\right)\right| \leq 2^{r} M\left(a_{j}\right)$, (1) and (2) of Corollary 3.4 are satisfied; it remains to verify (3). First, fix $j \geq 1, l \geq 1$, and $k_{j-l}, \ldots, k_{j+l} \in \mathbf{N}^{*}$ and take $\omega, \omega^{\prime} \in \Delta:=\Delta_{j l}\left(k_{j-l}, \ldots, k_{j+l}\right)$ where

$$
\Delta_{j l}\left(k_{j-l}, \ldots, k_{j+l}\right)= \begin{cases}\left\{a_{j-l}=k_{j-l}, \ldots, a_{j+l}=k_{j+l}\right\} & \text { if } j-l \geq 1, \\ \left\{a_{1}=k_{1}, \ldots, a_{j+l}=k_{j+l}\right\} & \text { if } j-l<1\end{cases}
$$

we claim that

$$
\left|u_{j}(\omega)-u_{j}\left(\omega^{\prime}\right)\right|<62^{-l}
$$

We have

$$
\begin{aligned}
\left|x_{j}(\omega)-x_{j}\left(\omega^{\prime}\right)\right| & =\left|a_{j}(\omega)+x_{j+1}(\omega)^{-1}-\left(a_{j}\left(\omega^{\prime}\right)+x_{j+1}\left(\omega^{\prime}\right)^{-1}\right)\right| \\
& =\left|x_{j+1}(\omega)^{-1}-x_{j+1}\left(\omega^{\prime}\right)^{-1}\right|<22^{-1}
\end{aligned}
$$

because $x_{j+1}(\omega)^{-1}=\left[0, k_{j+1}, \ldots, k_{j+1}, a_{j+l+1}(\omega), \ldots\right]$ and $x_{j+1}\left(\omega^{\prime}\right)^{-1}=$ $\left[0, k_{j+1}, \ldots, k_{j+l}, a_{j+l+1}\left(\omega^{\prime}\right), \ldots\right]$ both are in the fundamental interval of rank $l,\left\{\alpha \in[0,1): a_{1}(\alpha)=k_{j+1}, \ldots, a_{l}(\alpha)=k_{j+l}\right\}$ whose length is less than $2^{-(l-1)}$. This proves (4.4) when $j=1$. Now suppose $j \geq 2$ and recall that $u_{j}=x_{j}+\left(y_{j-1}\right)^{-1}$. If $j-l \leq 1$ we have $y_{j-1}(\omega)^{-1}=\left[0, k_{j-1}, \ldots, k_{1}\right]=$ $y_{j-1}\left(\omega^{\prime}\right)^{-1}$ and (4.4) holds. Suppose $j-l \geq 2$. If $l=1$ merely observe that $\left|y_{j-1}(\omega)^{-1}-y_{j-1}\left(\omega^{\prime}\right)^{-1}\right|<1<42^{-l}$. If $l \geq 2$ then, writing

$$
\tilde{\omega}=y_{j-1}(\omega)^{-1}=\left[0, k_{j-1}, \ldots, k_{j-1}, a_{j-l-1}(\omega), \ldots, a_{1}(\omega)\right]
$$

and

$$
\tilde{\omega}^{\prime}=y_{j-1}\left(\omega^{\prime}\right)^{-1}=\left[0, k_{j-1}, \ldots, k_{j-1}, a_{j-1-1}\left(\omega^{\prime}\right), \ldots, a_{1}\left(\omega^{\prime}\right)\right],
$$


we conclude that

$$
a_{0}(\tilde{\omega})=0=a_{0}\left(\tilde{\omega}^{\prime}\right), \quad a_{i}(\tilde{\omega})=k_{j-i}=a_{i}\left(\tilde{\omega}^{\prime}\right) \quad \text { if } 1 \leq i \leq l-1
$$

(we have used the following fact: if $\alpha=\left[k_{0}, \ldots, k_{N}\right]$ with $k_{0} \in \mathbf{Z}, k_{1}, \ldots, k_{N}$ $\in \mathbf{N}^{*}$ and $N \geq 2$ then $a_{i}(\alpha)=k_{i}$ if $\left.0 \leq i \leq N-2\right)$. Thus $\tilde{\omega}$ and $\tilde{\omega}^{\prime}$ both belong to $\left\{\alpha \in[0,1): a_{1}(\alpha)=k_{j-1}, \ldots, a_{l-1}(\alpha)=k_{j-(l-1)}\right\}$ whose length is $<2^{-(l-2)}$ Therefore $\left|y_{j-1}(\omega)^{-1}-y_{j-1}\left(\omega^{\prime}\right)^{-1}\right|<42^{-l}$ and (4.4) holds. By (4.2) we obtain

$$
\left|\eta_{j}(\omega)-\eta_{j}\left(\omega^{\prime}\right)\right| \leq 6^{r}\left(2^{r}\right)^{-l} M\left(a_{j}(\omega)\right) ;
$$

this implies

$$
\begin{aligned}
\left|\eta_{j}(\omega)-\frac{1}{P(\Delta)} \int_{\Delta} \eta_{j} d P\right| & =\left|\frac{1}{P(\Delta)} \int_{\Delta}\left(\eta_{j}(\omega)-\eta_{j}\right) d P\right| \\
& \leq 6^{r}\left(2^{r}\right)^{-l} M\left(a_{j}(\omega)\right) .
\end{aligned}
$$

Hence

$$
E_{P}^{1 / 2}\left(\eta_{j}-E_{P}\left(\eta_{j} \mid \mathscr{M}_{j l}\right)\right)^{2} \leq 6^{r}\left(2^{r}\right)^{-l} E_{P}^{1 / 2} M^{2}\left(a_{1}\right)
$$

for every $l \geq 1, j \geq 1$ and (3) of Corollary 3.4 is verified.

4.4. Remarks. (a) In the proof of the preceding lemma, $\left\{x_{j}\right\}$ case, Corollary 3.4 can be replaced by the (functional version of the) theorem in [5, p. 192] (consider the function $\tilde{f}(\omega)=f\left(\omega^{-1}\right)-f\left(\left[\omega^{-1}\right]\right)$ ).

(b) Let $\alpha \geq 1 / 2$ and $c>0$. The function

$$
f(x)=x^{\alpha}\left(c-(\log x)^{-1 / 2} \cos \left((\pi / 3)^{x-[x]}\right)\right)
$$

is regularly varying with exponent $\alpha$ but does not satisfy (4.2) (for some $b>0$, $f^{\prime}(x)>b x^{\alpha}(\log x)^{-1 / 2}$ if $x \in(k, k+1)$ and $k \geq 1$; then $E_{P}\left(f\left(x_{1}\right)-f\left(a_{1}\right)\right)^{2}=$ $\infty)$. Hence $\left(K_{0}\right)$ below is not satisfied; we do not know whether the law of $\sum_{1}^{n} f\left(x_{j}\right)$, suitable normalized, converges.

For $x_{j}$ we have $\mathscr{L}_{P}\left(x_{j}\right)(d t)=I_{(1, \infty)}(t)(t(t+1) \log 2)^{-1} d t$ for every $j$. For $u_{j}$ the next result is useful. (4.6) is proved in [7, p. 365] and (4.7) is (apart from the specification of $r$ ) a reformulation of the theorem in [15]; by (a) both are consequences of a result of Lévy. Our proof follows an indication in [7].

4.5. Lemma. Denote $G_{n}(t)=\lambda\left(y_{n}>t\right)$ for real $t$ and $n \geq 1$.

(a) If $n \geq 2$ then

$$
H_{n}(t):=\lambda\left(u_{n} \leq t\right)= \begin{cases}\frac{1}{t} \int_{0}^{t-1} G_{n-1}\left(\frac{1}{s}\right) d s & \text { if } t \geq 1, \\ 0 & \text { if } t<1,\end{cases}
$$

and

$$
h_{n}(t)=I_{(1, \infty)}(t)\left\{t^{-1} G_{n-1}\left((t-1)^{-1}\right)-t^{-2} \int_{0}^{t-1} G_{n-1}\left(s^{-1}\right) d s\right\}
$$


is a density function for $\mathscr{L}_{\lambda}\left(u_{n}\right)$; moreover $H_{n}(t)=1-t^{-1}\left(1+E_{\lambda}\left(1 / y_{n-1}\right)\right)$ if $t \geq 2$ and $h_{n}(t)=t^{-2}\left(1+E_{\lambda}\left(1 / y_{n-1}\right)\right)$ if $t>2$.

(b) Let $H$ be the distribution function with density

$$
h(t)=(\log 2)^{-1}\left\{I_{(1,2]}(t) t^{-1}\left(1-t^{-1}\right)+I_{(2, \infty)}(t) t^{-2}\right\} .
$$

Then there exists $r \in(0,1)$ such that

$$
\begin{aligned}
& \sup _{t}\left|H_{n}(t)-H(t)\right|=O\left(r^{n}\right), \\
& \sup _{t}\left|h_{n}(t)-h(t)\right|=O\left(r^{n}\right) .
\end{aligned}
$$

Proof. (a) Let $n \geq 2$. By Proposition 2.1 we have if $1<t \leq 2$

$$
\begin{aligned}
H_{n}(t) & =\sum_{y} \lambda\left(x_{n}+\frac{1}{y_{n-1}} \leq t \mid y_{n-1}=y\right) \lambda\left(y_{n-1}=y\right) \\
& =\sum_{y: y>(t-1)^{-1}} \lambda\left(1<x_{n} \leq t-y^{-1} \mid y_{n-1}=y\right) \lambda\left(y_{n-1}=y\right) \\
& =\sum_{y: y>(t-1)^{-1}}\left(1-t^{-1}-t^{-1} y^{-1}\right) \lambda\left(y_{n-1}=y\right) \\
& =\left(1-t^{-1}\right) \lambda\left(y_{n-1}>(t-1)^{-1}\right)-t^{-1} E_{\lambda}\left(\frac{1}{y_{n-1}} ; y_{n-1}>(t-1)^{-1}\right)
\end{aligned}
$$

and if $t>2$

$$
\begin{aligned}
\lambda\left(2<u_{n} \leq t\right) & =\sum_{y} \lambda\left(2-y^{-1}<x_{n} \leq t-y^{-1} \mid y_{n-1}=y\right) \lambda\left(y_{n-1}=y\right) \\
& =\left(2^{-1}-t^{-1}\right) \sum_{y}\left(1+y^{-1}\right) \lambda\left(y_{n-1}=y\right) \\
& =\left(2^{-1}-t^{-1}\right)\left(1+E_{\lambda}\left(1 / y_{n-1}\right)\right) .
\end{aligned}
$$

But an integration by parts shows that

$E_{\lambda}\left(\frac{1}{y_{n-1}} ; \frac{1}{y_{n-1}}<t-1\right)=(t-1) \lambda\left(\frac{1}{y_{n-1}}<t-1\right)-\int_{0}^{t-1} \lambda\left(\frac{1}{y_{n-1}}<s\right) d s$ if $t \geq 1$, which implies (since $y_{n-1} \geq 1$ )

$$
E_{\lambda}\left(\frac{1}{y_{n-1}}\right)=1-\int_{0}^{1} \lambda\left(\frac{1}{y_{n-1}}<s\right) d s .
$$

From the preceding relations we can easily obtain the indicated expressions for $H_{n}$. The property of $h_{n}$ follows from the equality

$$
\begin{aligned}
\int_{1}^{u} & \left(\int_{0}^{t-1} t^{-2} \lambda\left(\frac{1}{y_{n-1}}<s\right) d s\right) d t \\
& =\int_{1}^{u} \frac{1}{t} \lambda\left(\frac{1}{y_{n-1}}<t-1\right) d t-\frac{1}{u} \int_{0}^{u-1} \lambda\left(\frac{1}{y_{n-1}}<s\right) d s
\end{aligned}
$$


where $u>1$. On the other hand, note that

$$
h_{n}(t)=t^{-2}\left(2-\int_{0}^{1} \lambda\left(y_{n-1}>s^{-1}\right) d s\right) \quad \text { if } t>2 .
$$

(b) It is proved in [19, Chapitre IX] that the function

$$
\begin{aligned}
F(x): & =(\log 2)^{-1} \log (2 x /(x+1)) \quad \text { if } x>1, \\
& =0 \text { if } x \leq 1
\end{aligned}
$$

satisfies

$$
\sup _{x}\left|\lambda\left(y_{n} \leq x\right)-F(x)\right| \leq C r^{n}
$$

for some $C>0$ and $r \in(0,1)$. Now (4.6) and (4.7) follow from (a) since $H$ and $h$ are related to $1-F$ just as $H_{n}$ and $h_{n}$ are to $G_{n-1}$.

4.6. Corollary. Assume $f:[1, \infty) \rightarrow(0, \infty)$ is regularly varying with exponent $\alpha \in[1 / 2,1], E_{P} f^{2}\left(a_{1}\right)=+\infty$ and satisfies

$$
\begin{aligned}
& f(x)=x^{\alpha} L(x) \text { where } L(x)=c \exp \left\{\int_{1}^{x} \varepsilon(t) t^{-1} d t\right\} \text { with } c>0, \\
& \varepsilon:[1, \infty) \rightarrow \mathbf{R} \text { measurable, bounded and } \lim _{t \rightarrow \infty} \varepsilon(t)=0 .
\end{aligned}
$$

Let $\nu_{\alpha}$ be defined by $(2.14)$ if $\alpha \in(1 / 2,1]$ and write $\nu_{1 / 2}=N(0,1)$. Let

$$
m(f)=(\log 2)^{-1} \int_{1}^{\infty} \int_{1}^{\infty}\left(f\left(x+y^{-1}\right)-f([x])\right)(x y+1)^{-2} d x d y
$$

if $\alpha=1, m(f)=\int_{1}^{\infty} f(t) h(t) d t$ ( $h$ being the density in (4.5)) if $\alpha \in[1 / 2,1$ ) and define $\xi_{n}$ by

$$
\xi_{n}(t)=f(n)^{-1} \sum_{1 \leq j \leq[n t]}\left\{f\left(u_{j}\right)-m(f)-E_{P}\left(f\left(a_{1}\right) ; f\left(a_{1}\right) \leq f(n)\right)\right\} \quad \text { if } \alpha=1,
$$

$$
\begin{aligned}
& \xi_{n}(t)=f(n)^{-1} \sum_{1 \leq j \leq[n t]}\left\{f\left(u_{j}\right)-m(f)\right\} \quad \text { if } \alpha \in(1 / 2,1), \\
& \xi_{n}(t)=b(n)^{-1} \sum_{1 \leq j \leq[n t]}\left\{f\left(u_{j}\right)-m(f)\right\} \quad \text { if } \alpha=1 / 2,
\end{aligned}
$$

where $\{b(n)\}$ is any sequence satisfying $\lim _{n} n b(n)^{-2} \widetilde{U}(b(n))=1$ (with $\widetilde{U}$ defined as in Corollary 2.12). Then for any $\rho \ll \lambda, \mathscr{L}_{\rho}\left(\xi_{n}\right) \underset{w}{\rightarrow} Q_{\nu_{a}}$. The same result holds if $\xi_{n}$ is defined by replacing in (4.9)- (4.11) $u_{j}$ by $x_{j}$ and $m(f)$ by $m^{\prime}(f)$ where $m^{\prime}(f)=E_{P}\left(f\left(x_{1}\right)-f\left(a_{1}\right)\right)$ if $\alpha=1,=E_{P} f\left(x_{1}\right)$ if $\alpha \in[1 / 2,1)$. Proof. By $\left(K_{0}\right) f$ is bounded on finite intervals and, as we will show, it satisfies (4.2). Writing $M=\max \left\{1, \sup _{t \geq 1}|\varepsilon(t)|\right\}$, by $\left(K_{0}\right)$ we have if $k \in \mathbf{N}^{*}, k \leq$ $x<y \leq k+2$,

$$
|L(x)-L(y)| \leq L(x)\left((y / x)^{M}-1\right) \leq M^{\prime} L(x) x^{-1}|x-y|
$$


where $M^{\prime}=M 3^{M-1}\left(x^{-1} y<3\right)$; then, since $\alpha \leq 1$,

$$
\begin{aligned}
|f(x)-f(y)| & \leq x^{\alpha}|L(x)-L(y)|+\left|x^{\alpha}-y^{\alpha}\right| L(y) \\
& \leq M^{\prime}(L(x)+L(y))|x-y| .
\end{aligned}
$$

On the other hand, there exists $C$ such that $L(x) \leq C x^{1 / 4}$ for every $x \geq 1$. Thus if $k \in \mathbf{N}^{*}$ and $x, y \in[k, k+2]$

$$
|f(x)-f(y)| \leq M^{\prime} 2 C(3 k)^{1 / 4}|x-y|=M(k)|x-y| \quad \text { (say) }
$$

which proves (4.2). Corollaries 2.12 and 2.13 and Lemma 4.3 now imply the assertion about $\left\{x_{j}\right\}$. For $\left\{u_{j}\right\}$ we conclude that $\mathscr{L}_{\rho}\left(\xi_{n}^{\prime}\right) \rightarrow_{w} Q_{\nu_{a}}, \xi_{n}^{\prime}$ being defined by (4.9) with $m(f)$ replaced by $E_{P}\left(f\left(u_{j}\right)-f\left(a_{j}\right)\right)$ (which depends on $j)$ in the case $\alpha=1$ and by (4.10)-(4.11) with $m(f)$ replaced by $E_{P} f\left(u_{j}\right)$ if $\alpha \in[1 / 2,1)$.

Suppose $\alpha \in[1 / 2,1)$. By Lemma 4.5 we have

$$
\begin{aligned}
\left|E_{\lambda} f\left(u_{n}\right)-m(f)\right| \leq & \left(\sup _{t \in[1,2]} f(t)\right) \sup _{t}\left|h_{n}(t)-h(t)\right| \\
& +\left(\int_{2}^{\infty} f(t) t^{-2} d t\right)\left|1+E_{\lambda}\left(\frac{1}{y_{n-1}}\right)-\frac{1}{\log 2}\right|
\end{aligned}
$$

and hence, for some constant $C_{1}$,

$$
\left|E_{\lambda} f\left(u_{n}\right)-m(f)\right| \leq C_{1} r^{n} \quad \text { for every } n \geq 1 .
$$

Write $g_{n l}=E_{P}\left(f\left(u_{n}\right) \mid \mathscr{M}_{n l}\right)$. As in the proof of Lemma 4.3, using (4.4) and (4.12), we obtain that for some $C_{2}$

$$
E_{P}^{1 / 2}\left(f\left(u_{n}\right)-g_{n l}\right)^{2} \leq C_{2} 2^{-l} \text { for every } n \geq 1 \text { and } l \geq 1 \text {. }
$$

On the other hand, since there exist constants $K$ and $r^{\prime} \in(0,1)$ such that $|P(A)-\lambda(A)| \leq K\left(r^{\prime}\right)^{k} P(A)$ for any $A \in \sigma\left(a_{k}, a_{k+1}, \ldots\right), k \geq 1$ (argue as in the proof of [12, Lemma 19.4.2] using (7) of [20]), we have for some $C_{3}$

$$
\left|E_{P} g_{n l}-E_{i} g_{n l}\right| \leq C_{3}\left(r^{\prime}\right)^{n-l} \text { if } n>l \geq 1
$$

$\left(\left|\int_{0}^{\infty}\left(P\left(g_{n l}>x\right)-\lambda\left(g_{n l}>x\right)\right) d x\right| \leq K E_{P} f\left(u_{n}\right)\left(r^{\prime}\right)^{n-l}\right)$. Taking $l_{n}=[n / 2]$ we get from $(4.13)-(4.15)$

$$
\left|E_{P} f\left(u_{n}\right)-m(f)\right| \leq C_{2} 2^{-l_{n}}+C_{3}\left(r^{\prime}\right)^{n-l_{n}}+(2 \log 2)^{1 / 2} C_{2} 2^{-l_{n}}+C_{1} r^{n} .
$$

Thus $\left|E_{P} f\left(u_{n}\right)-m(f)\right|=O\left(s^{n}\right)$ for some $s \in(0,1)$ which implies that $\sup _{t}\left|\xi_{n}(t)-\xi_{n}^{\prime}(t)\right| \rightarrow 0$ pointwise and so the proof in the case $\alpha<1$ is complete.

Now assume $\alpha=1$. First observe that Proposition 2.1 implies that for any Borel measurable function $h$

$$
\int_{\left\{y_{n-1}=y\right\}} h\left(x_{n}\right) d \lambda=\left(\int_{1}^{\infty} h(x) \frac{y(y+1)}{(x y+1)^{2}} d x\right) \lambda\left(y_{n-1}=y\right)
$$


provided one of the two members exists, $y$ being a possible value of $y_{n-1}$. Thus, writing

$$
K(y)=\int_{1}^{\infty}\left(f\left(x+y^{-1}\right)-f([x])\right) y(y+1)(x y+1)^{-2} d x, \quad y \geq 1,
$$

we have (by (4.12) $K$ is bounded and the following integrals exist)

$$
\begin{aligned}
E_{\lambda}\left(f\left(u_{n}\right)-f\left(a_{n}\right)\right) & =\sum_{y} \int_{\left\{y_{n-1}=y\right\}}\left(f\left(x_{n}+\frac{1}{y}\right)-f\left(\left[x_{n}\right]\right)\right) d \lambda \\
& =\int_{[1, \infty)} K d \mathscr{L}_{\lambda}\left(y_{n-1}\right) .
\end{aligned}
$$

On the other hand, $m(f)=\int_{[1, \infty)} K d F$ where $F$ is the distribution function appearing in (4.8).

Denote $g(x, y)$ the integrand in (4.16) and $v(x, y)=y(y+1)(x y+1)^{-2}$. If $x \geq 1$ and $y^{\prime}>y \geq 1$ we get by (4.12)

$$
\begin{aligned}
\left|g(x, y)-g\left(x, y^{\prime}\right)\right| \leq & M([x])\left|y^{-1}-\left(y^{\prime}\right)^{-1}\right||v(x, y)| \\
& +M([x])\left|x+\left(y^{\prime}\right)^{-1}-[x]\right|\left|v(x, y)-v\left(x, y^{\prime}\right)\right| \\
\leq & 10 M([x])(x y)^{-2}\left|y-y^{\prime}\right| .
\end{aligned}
$$

Hence if $y^{\prime}>y \geq 1$ we have

$$
\begin{aligned}
\left|K(y)-K\left(y^{\prime}\right)\right| & \leq 10\left(\int_{1}^{\infty} M([x]) x^{-2} d x\right) y^{-2}\left|y-y^{\prime}\right| \\
& =A y^{-2}\left|y-y^{\prime}\right| \quad(\text { say })
\end{aligned}
$$

and $K$ is absolutely continuous. Then.

$$
\left|E_{\lambda}\left(f\left(u_{n}\right)-f\left(a_{n}\right)\right)-m(f)\right| \leq A \int_{1}^{\infty}\left|\lambda\left(y_{n-1}>t\right)-(1-F(t))\right| t^{-2} d t
$$

and (4.8) gives that $\left|E_{\lambda}\left(f\left(u_{n}\right)-f\left(a_{n}\right)\right)-m(f)\right|=O\left(r^{n}\right)$. In order to complete the proof, observe that analogous relations to (4.14) and (4.15) are valid and argue as above.

\subsection{Examples.}

4.7.1. If $f(x)=x^{\alpha}$ where $\alpha \in[1 / 2,1)$ then

$$
m(f)=(\alpha(1-\alpha) \log 2)^{-1}\left(2^{\alpha}-1\right)
$$

and we can take $b(n)=(n \log n / \log 2)^{1 / 2}$ in (4.11).

4.7.2. Let $f(x)=x$. Then $m^{\prime}(f)=(\log 2)^{-1}-1$ and $m(f)=m^{\prime}(f)+$ $(\log 2)^{-1} \int_{1}^{\infty} y^{-2}(y+1)^{-1} d y=2\left((\log 2)^{-1}-1\right)$. If $\xi_{n}$ is defined by $(2.20)$ then for any $\rho \ll \lambda, \mathscr{L}_{\rho}\left(\tilde{\xi}_{n}\right) \rightarrow_{w} Q_{\tilde{\nu}^{\prime}}$ and $\mathscr{L}_{\rho}\left(\tilde{\xi}_{n}\right) \rightarrow_{w}, Q_{\tilde{\nu}^{\prime}}$, where

$$
\tilde{\nu}^{\prime}=\delta_{\left((\log 2)^{-1}-1\right)} * \nu^{\prime}, \quad \tilde{\tilde{\nu}}^{\prime}=\delta_{2\left((\log 2)^{-1}-1\right)} * \nu^{\prime},
$$


$\nu^{\prime}$ being defined by (2.21) (we use the notation at the beginning of this section). Similar remarks to those made in 2.14.2 apply. We point out that the convergence of $\mathscr{L}_{\lambda}\left(\tilde{\tilde{\xi}}_{n}(1)\right)$ was indicated by Doeblin [7, p. 365].

Acknowledgment. The final draft of this paper was written during a visit to the Department of Mathematics and Statistics at Case Western Reserve University. I am grateful to this institution for its support and hospitality.

\section{REFERENCES}

1. A. de Acosta, Invariance principles in probability for triangular arrays of $B$-valued random vectors and some applications, Ann. Probab. 10 (1982), 346-373.

2. A. Araujo and E. Giné, The Central Limit Theorem for real and Banach valued random variables, Wiley, New York, 1980.

3. H. Bergström, On the convergence of sums of random variables in distribution under mixing condition, Period. Math. Hungar. 2 (1972), 173-190.

4. P. Billingsley, Ergodic theory and information, Wiley, New York, 1965.

5. __ Convergence of probability measures, Wiley, New York, 1968.

6. R. C. Bradley, Basic properties of strong mixing conditions. Dependence in probability and statistics (E. Eberlein and M. S. Taqqu, eds.), Prog. Prob. Statist., vol. 11, Birkhäuser, Boston, Mass., 1986.

7. W. Doeblin, Remarques sur la théorie métrique des fractions continues, Compositio Math. 7 (1940), 353-371.

8. J. Galambos, The distribution of the largest coefficient in continued fraction expansions, Quart. J. Math. Oxford Ser. (2) 23 (1972), 147-151.

9. I. I. Gihman and A. V. Skorohod, The theory of stochastic processes. II, Springer-Verlag, New York, 1974.

10. G. H. Hardy and E. M. Wright, An introduction to the theory of numbers, Clarendon Press, Oxford, 1960.

11. I. A. Ibragimov, Some limit theorems for stationary processes, Theory Probab. Appl. 7 (1962), 349-382.

12. I. A. Ibragimov and Yu. V. Linnik, Independent and stationary sequences of random variables, Wolters-Noordhoff, Groningen, 1971.

13. M. Iosifescu, A Poisson law for $\psi$-mixing sequences establishing the truth of a Doeblin's statement, Rev. Roumaine Math. Pures Appl. 22 (1977), 1441-1447.

14. A. Ya. Khintchine, Metrische Kettenbruchprobleme, Compositio Math. 1 (1935), 361-382.

15. D. E. Knuth, The distribution of continued fraction approximations, J. Number Theory 19 (1984), 443-448.

16. H. A. Krieger, A new look at Bergström's theorem on convergence in distribution for sums of dependent random variables, Israel J. Math. 47 (1984), 32-64.

17. S. Lang, Introduction to Diophantine approximations, Addison-Wesley, Reading, Mass., 1966.

18. P. Lévy, Fractions continues aléatoires, Rend. Circ. Mat. Palermo 1 (1952), 170-208.

19. _. Théorie de l'addition des variables aléatoires, Gauthier-Villars, Paris, 1954.

20. W. Philipp, Some metrical theorems in number theory. II, Duke Math. J. 37 (1970), 447-458.

21. Mixing sequences of random variables and probabilistic number theory. Mem. Amer. Math. Soc. No. 114, 1971.

22. Weak and $L^{p}$-invariance principles for sums of $B$-ralued random variables, Ann. Probab. 8 (1980), 68-82. Correction ihid. 14 (1986), 1095-1101.

23. L Limit theorems for sums of partial quotients of continued fractions, Monatsh. Math. 105 (1988), 195-206. 
24. J. D. Samur, Convergence of sums of mixing triangular arrays of random vectors with stationary rows, Ann. Probab. 12 (1984), 390-426.

25. _ A note on the convergence to Gaussian laws of sums of stationary $\phi$-mixing triangular arrays, Proc. Probability in Banach Spaces. V, Lecture Notes in Math., vol. 1153, SpringerVerlag, Berlin, 1985, pp. 387-399.

26. __ On the invariance principle for stationary $\phi$-mixing triangular arrays with infinitely divisible limits, Probab. Theory Related Fields 75 (1987), 245-259.

27. E. Seneta, Regularly varying functions, Lecture Notes in Math., vol. 508, Springer-Verlag, Berlin, 1976.

Departamento de Matemática, Facultad de Ciencias Exactas, Universidad Nacional de la Plata, Casilla de Correo 172, 1900 la Plata, Argentina 